\title{
Integrated Guidance and Control of Interceptor Missile Based on Asymmetric Barrier Lyapunov Function
}

\author{
Xiang Liu (D) and Xiaogeng Liang \\ School of Automation, Northwestern Polytechnical University, Xi'an 710072, China \\ Correspondence should be addressed to Xiang Liu; 1x861002@163.com
}

Received 29 July 2018; Accepted 13 February 2019; Published 28 April 2019

Academic Editor: Zhiguang Song

Copyright (c) 2019 Xiang Liu and Xiaogeng Liang. This is an open access article distributed under the Creative Commons Attribution License, which permits unrestricted use, distribution, and reproduction in any medium, provided the original work is properly cited.

In this study, a novel integrated guidance and control (IGC) algorithm based on an IGC method and the asymmetric barrier Lyapunov function is designed; this algorithm is designed for the interceptor missile which uses a direct-force/aerodynamicforce control scheme. First, by considering the coupling between the pitch and the yaw channels of the interceptor missile, an IGC model of these channels is established, and a time-varying gain extended state observer (TVGESO) is designed to estimate unknown interferences in the model. Second, by considering the system output constraint problem, an asymmetric barrier Lyapunov function and a dynamic surface sliding-mode control method are employed to design the control law of the pitch and yaw channels to obtain the desired control moments. Finally, in light of redundancy in such actuators as aerodynamic rudders and jet devices, a dynamic control allocation algorithm is designed to assign the desired control moments to the actuators. Moreover, the results of simulations show that the IGC algorithm based on the asymmetric barrier Lyapunov function for the interceptor missile allows the outputs to meet the constraints and improves the stability of the control system of the interceptor missile.

\section{Introduction}

The interceptor missile plays an important role in modern anti-missile systems. Given the rapid development of hypersonic aircraft, demands on accurate guidance and control of the interceptor missile has become increasingly stringent. To cope with high-speed aircraft with a strong penetration capability, the interceptor missile generally adopts a directforce/aerodynamic-force control scheme that can accelerate the speed of command response. The guidance and control system of the interceptor missile is a highly dynamic and multivariate system of strong coupling, rapid temporal change, and uncertainty, thereby making it critical to the examination of the guidance and control of interceptor missile at high speed with strict control requirements.

Integrated guidance and control (IGC) refers to using the relative interceptor-target motion information and the dynamic information of the interceptor missile to generate a control force that drives it to strike the target. IGC allows for a rational allocation of interceptor missile control capability to not only maintain the interceptor missile's flight attitude but also improve the precision of its guidance $[1,2]$. The authors in $[3,4]$ explored the IGC control law by using high-order sliding-mode control methods and backstepping control algorithms. On the basis of backstepping control algorithms, the authors in $[5,6]$ considered saturation factors and introduced dynamic models of actuators models to design algorithms for longitudinal and anti-saturation IGC for aircraft. An adaptive sliding-mode control method was used to implement the design of an IGC system on the pitch plane to configure missiles to attack ground targets [7], but the problem of target mobility was not considered. Based on a backstepping method, the missile control loop was regarded as a second-order element in the study [8], which introduced a first-order integral filter to estimate the derivative of the input to the virtual control and design the control law. Based on a three-dimensional (3D) ICG model, a robust adaptive backstepping method was implemented to design an IGC algorithm for a missile [9]. Methods of IGC design have been widely used in guidance and control systems of 
aircraft [10], missiles [11-13], and unmanned aerial vehicles $[14,15]$. In recent years, researchers have combined this method with modern control theories, such as dynamic surface control [16], optimal control [17], and predictive control [18], to generate methods of IGC design for aircrafts. However, most prevalent IGC design methods do not consider the coupling relationship between the pitch and the yaw channels. Moreover, to improve the stability of the guidance process, constraints concerning the angular velocities of the line of sight during the guidance of the interceptor missile should be considered.

Nonlinearity commonly exists in the IGC system of the interceptor missile. In recent years, with practical engineering problems' increasing demand on the control performance, there has been considerable progress in the development of nonlinear control theory, especially in adaptive control [19], neural network control [20], fuzzy control [21], etc. All of these have laid a solid foundation for the in-depth research of nonlinear control theory. However, in actual engineering applications, the use of nonlinear systems is always subject to input, output, and state constraints, among others, and violation of these constraints can result in the control system's downgraded performance. Therefore, it has now become an important research direction, when constructing the control system to consider the effect of these constraints and to properly handle them in the controller design process. The authors in [22] proposed an adaptive neural network constrained control algorithm for singleinput/single-output nonlinear stochastic switching systems; this algorithm constructed traditional Lyapunov function to handle constraint control, which achieved good results. As barrier Lyapunov function does not require an exact solution of the system, the constrained control method based on barrier Lyapunov function has been widely used in state constraint and output constraint problems in recent years. Barrier Lyapunov function is a special type of continuous function, unlike traditional Lyapunov function which is radially unbounded, in barrier Lyapunov function, when the parameters approach the limit value, the function value will tend to infinity to ensure that the control system satisfies the constraints [23]. Barrier Lyapunov function can be utilized to satisfy the constraints of both symmetric and asymmetric constraint controls, even when the constraint is a time-varying asymmetric one. According to the authors in [24], barrier Lyapunov function has been used to solve constraint problems in a hybrid PDE-ODE system that describes a nonuniform gantry crane system. The authors in [25] proposed an adaptive fuzzy neural network control method using impedance learning for a constrained robot system based on barrier Lyapunov function. According to the authors in [26, 27], barrier Lyapunov functions have been used to solve constraint problems in nonlinear and uncertain systems and to expand the definitions of the constraints. Methods based on these functions can effectively solve problems of symmetrically and asymmetrically constrained control. The authors in $[28,29]$ have expanded output control constraints to include time-varying outputs while relaxing the limitations on the initial values of control systems. By combining barrier Lyapunov functions with dynamic surface control technologies, some studies $[30,31]$ have proposed barrier Lyapunov function-based methods suitable for constrained dynamic surface control to solve the computational inflation problem caused by backstepping control. Researchers subsequently applied this method to brushless DC motors [32], plane braking systems [33], and hypersonic aircraft $[34,35]$ to achieve satisfactory results in terms of constrained control. However, few studies have investigated the application of this method to interceptor missile control. Design methods based on barrier Lyapunov functions are advantageous because they can solve the output constraint problem of interceptor missile guidance control systems and improve their stability.

In view of the above analysis, to aim at the interceptor missile which uses a direct-force/aerodynamic-force control scheme, and to consider the coupling relationship between the pitch and the yaw channels as well as the constraints on the system's output, this study proposes an IGC algorithm based on the asymmetric barrier Lyapunov function. First, a time-varying gain extended state observer (TVGESO) is designed to estimate interferences in the system. Second, an asymmetric barrier Lyapunov function and a dynamic surface sliding-mode control method, respectively, are used to design control laws for the interceptor missile to obtain the desired moments. Finally, a dynamic control allocation algorithm is designed to allocate the desired control moments. The results of simulations show that the proposed algorithm enables the outputs to meet the constraints and improves the stability of the interceptor missile control systems.

\section{IGC Model of Interceptor Missile}

The relationship of relative motion between the interceptor missile and its target in 3D space is shown in Figure 1.

In the figure, Oxyz refers to an inertial coordinate system and $O x_{4} y_{4} z_{4}$ refers to a line-of-sight coordinate system, respectively; $M$ and $T$ refer to the interceptor missile and the target, respectively; $q_{\varepsilon}$ and $q_{\beta}$ are the vertical and horizontal angles of the line of sight with the interceptor missile and the target, respectively, and $r$ denotes the relative distance between the interceptor missile and the target. The model of the relative motion of the interceptor missile and target is as follows:

$$
\left\{\begin{array}{l}
r \ddot{q}_{\varepsilon}=-2 \dot{r} \dot{q}_{\varepsilon}-r \dot{q}_{\beta}^{2} \sin q_{\varepsilon} \cos q_{\varepsilon}-a_{t \varepsilon}-a_{m 4 \varepsilon}, \\
\ddot{r}=r \dot{q}_{\varepsilon}^{2}+r \dot{q}_{\beta}^{2} \cos ^{2} q_{\varepsilon}+a_{t r}-a_{m 4 r}, \\
-r \ddot{q}_{\beta} \cos q_{\varepsilon}=2 \dot{r} \dot{q}_{\beta} \cos q_{\varepsilon}-2 r \dot{q}_{\varepsilon} \dot{q}_{\beta} \sin q_{\varepsilon}+a_{t \beta}-a_{m 4 \beta},
\end{array}\right.
$$

where $\dot{q}_{\varepsilon}$ and $\dot{q}_{\beta}$ denote the vertical and horizontal angular velocities of the line of sight with the interceptor missile and the target, respectively; $a_{m 4 \varepsilon}$ and $a_{m 4 \beta}$ denote the longitudinal and lateral accelerations of the interceptor missile, respectively; and $a_{t \varepsilon}$ and $a_{t \beta}$ denote the longitudinal and lateral accelerations of the target, respectively. 


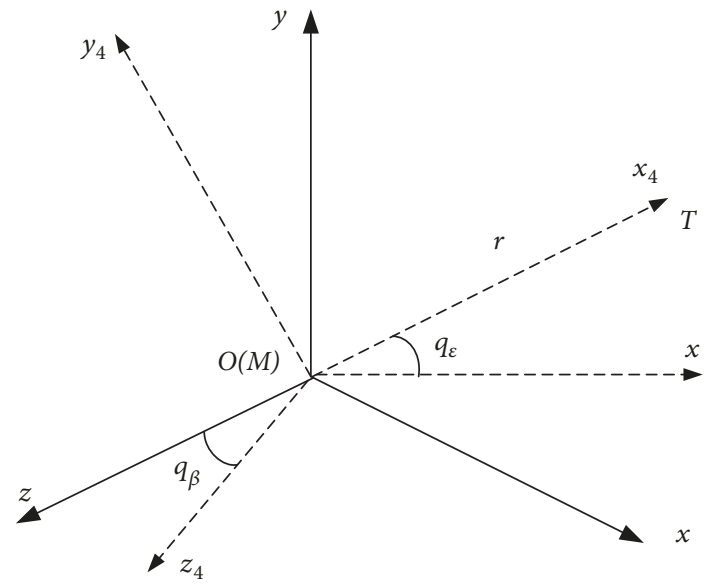

Figure 1: The relative relationship between the interceptor missile and its target in $3 \mathrm{D}$ space.

The interceptor missile uses a direct-force/aerodynamicforce control scheme. Assuming that the direct force is adjustable and continuous, the angles of deflection of the rudder equivalent to the direct force in the pitch and the yaw channels are, respectively, defined as

$$
\begin{aligned}
& \delta_{s y}=\frac{F_{y}}{F_{s \max }}, \\
& \delta_{s z}=\frac{F_{z}}{F_{s \max }},
\end{aligned}
$$

where $F_{z}$ and $F_{y}$ denote the thrust generated by the jet device, and $F_{s \max }$ is the maximum steady-state thrust of the jet devices.

In light of the coupling relationship between the pitch and yaw channels of the interceptor missile, its dynamic model is expressed as follows:

$$
\left\{\begin{array}{l}
\dot{\alpha}=\omega_{z}+\omega_{y} \tan \beta \sin \alpha-\frac{q S C_{y}^{\alpha} \alpha \cos \alpha}{m V_{m} \cos \beta}+d_{\alpha} \\
\dot{\beta}=\omega_{y} \cos \alpha+\frac{q S C_{y}^{\alpha} \alpha \sin \alpha \sin \beta}{m V_{m}}+\frac{q S C_{z}^{\beta} \beta \cos \beta}{m V_{m}}+d_{\beta} \\
\dot{\omega}_{z}=\frac{q S \bar{L} m_{z}^{\alpha} \alpha}{J_{z}}+\frac{q S \bar{L} m_{z}^{\omega_{z}} \omega_{z}}{J_{z}}+\frac{q S \bar{L} m_{z}^{\delta_{z}} \delta_{z}}{J_{z}}+\frac{\left(1+K_{z}\right) F_{y} L_{m}}{J_{z}}+d_{\omega_{z}} \\
\dot{\omega}_{y}=\frac{q S \bar{L} m_{y}^{\beta} \beta}{J_{y}}+\frac{q S \bar{L} m_{y}^{\omega_{y}} \omega_{y}}{J_{y}}+\frac{q S \bar{L} m_{y}^{\delta_{y}} \delta_{y}}{J_{y}}+\frac{\left(1+K_{y}\right) F_{z} L_{m}}{J_{y}}+d_{\omega_{y}}
\end{array}\right.
$$

$$
\left\{\begin{array}{l}
a_{m 3 \varepsilon}=\frac{q S C_{y}^{\alpha} \alpha}{m}, \\
a_{m 3 \beta}=\frac{q S C_{z}^{\beta} \beta}{m},
\end{array}\right.
$$

where $S$ is the reference area of the interceptor missile; $q$ is the dynamic pressure; $V_{\mathrm{m}}$ is the speed of the interceptor missile; $\bar{L}$ is its reference length; $L_{\mathrm{m}}$ is the average distance between the jet device and its center of mass; $m$ is the mass of the interceptor missile; $\alpha$ and $\beta$ denote the attack angle and the sideslip angle, respectively; $\omega_{z}$ and $\omega_{y}$ denote the angular velocities of the pitch and the yaw, respectively; $\delta_{z}$ and $\delta_{y}$ denote the angles of deflection of the aerodynamic rudder; $K_{z}$ and $K_{y}$ denote the amplification factors of moment (used to describe the effect of the mutual interference between lateral jets and incoming flow on the aerodynamic moment of the interceptor missile); $d_{\alpha}, d_{\omega_{z}}, d_{\beta}$, and $d_{\omega_{y}}$ refer to the disturbances and uncertain interferences at each link of the system; $J_{z}$ and $J_{y}$ refer to the moments of inertia; $C_{y}^{\alpha}, C_{z}^{\beta}, m_{z}^{\alpha}$, $m_{z}^{\omega_{z}}, m_{z}^{\delta_{z}}, m_{y}^{\beta}, m_{y}^{\omega_{y}}$, and $m_{y}^{\delta_{y}}$ refer to the relevant aerodynamic forces and coefficients of moment; and $a_{m 3 \varepsilon}$ and $a_{m 3 \beta}$ refer to the vertical and lateral overloads of the interceptor missile, respectively.

Assuming that the angle of the line of sight of the interceptor missile in the terminal guidance stage changes slightly and that the angle of line of sight and direction of velocity of the interceptor missile are relatively small, let $a_{m 3 \varepsilon}=a_{m 4 \varepsilon}$ and $a_{m 3 \beta}=a_{m 4 \beta}$. According to Equations (1)-(4), by defining $x_{z 1}=q_{\varepsilon}, x_{z 2}=\dot{q}_{\varepsilon}, x_{z 3}=\alpha, x_{z 4}=\omega_{z}, x_{y 1}=q_{\beta}, x_{y 2}=\dot{q}_{\beta}, x_{y 3}=\beta$, and $x_{y 4}=\omega_{y}$, one can have the nonlinear IGC model in the pitch channel for the interceptor missile:

$$
\left\{\begin{array}{l}
\dot{x}_{z 2}=f_{z 2} x_{z 2}+g_{z 2} x_{z 3}-x_{y 2}^{2} \sin x_{z 1} \cos x_{z 1}+\frac{a_{t \varepsilon}}{r} \\
\dot{x}_{z 3}=f_{z 3} x_{z 3}+x_{z 4}+d_{z 3} \\
\dot{x}_{z 4}=f_{z 4} x_{z 3}+f_{z 5} x_{z 4}+g_{z 3} u_{z}+d_{z 4} \\
y_{z}=x_{z 2}
\end{array}\right.
$$

where $f_{z 2}=-(2 \dot{r} / r), f_{z 3}=-\left(q S C_{y}^{\alpha} / m V_{\mathrm{m}}\right), f_{z 4}=q S \bar{L} m_{z}^{\alpha} / J_{z}$, $f_{z 5}=q S \bar{L} m_{z}^{\omega_{z}} / J_{z}, g_{z 2}=-\left(q S C_{y}^{\alpha} / m r\right), g_{z 3}=1 / J_{z}, d_{z 3}=\omega_{y} \tan$ $\beta \sin \alpha+d_{\alpha}, d_{z 4}=d_{\omega_{z}}$, and $u_{z}=q S \bar{L} m_{z}^{\delta_{z}} \delta_{z}+\left(1+K_{z}\right) F_{y} L_{\mathrm{m}}$, with $u_{z}$ representing the moment generated jointly by both the aerodynamic rudders and the jet devices in the pitch channels.

Similarly, the nonlinear IGC model for the interceptor missile in its yaw channel is as follows:

$$
\left\{\begin{array}{l}
\dot{x}_{y 2}=f_{y 2} x_{y 2}+g_{y 2} x_{y 3}+2 x_{z 2} x_{y 2} \tan x_{z 1}-\frac{a_{t \beta}}{r \cos x_{z 1}} \\
\dot{x}_{y 3}=f_{y 3} x_{y 3}+x_{y 4}+d_{y 3} \\
\dot{x}_{y 4}=f_{y 4} x_{y 3}+f_{y 5} x_{y 4}+g_{y 3} u_{y}+d_{y 4} \\
y_{y}=x_{y 2}
\end{array}\right.
$$

where $f_{y 2}=-(2 \dot{r} / r), f_{y 3}=q S C_{y}^{\beta} / m V_{\mathrm{m}}, f_{y 4}=q S \bar{L} m_{y}^{\beta} / J_{y}, f_{y 5}=$ $q S \bar{L} m_{y}^{\omega_{y}} / J_{y}, g_{y 2}=q S C_{y}^{\beta} / m r \cos x_{z 1}, g_{y 3}=1 / J_{y}, d_{y 3}=\left(q S C_{y}^{\alpha} \alpha\right.$ $\left.\sin \alpha \sin \beta / m V_{m}\right)+d_{\beta}, d_{y 4}=d_{\omega_{y}}$, and $u_{y}=q S \bar{L} m_{y} \delta_{y} \delta_{y}+(1+$ $\left.K_{y}\right) F_{z} L_{m}$, with $u_{y}$ representing the moment generated jointly 
by both the aerodynamic rudders and the jet devices in the yaw channel.

Assumption 1. The unknown interferences $d_{z 3}, d_{z 4}, d_{y_{3}}$, and $d_{y 4}$ in the IGC models in Equations (5) and (6) of the interceptor missile are continuously differentiable, and the derivatives are bounded.

\section{Design of TVGESO}

A TVGESO can estimate nonlinear uncertainties in the model of the system and feed the estimates back into the control system for compensation. To eliminate the effects of unknown uncertain interferences $a_{t \varepsilon}, a_{t \beta}, d_{z 3}, d_{z 4}, d_{y 3}$, and $d_{y 4}$ in the system models in Equations (5) and (6) on the control system of the interceptor missile, a TVGESO is designed to estimate these interferences.

By defining $v_{r}=\dot{r}, v_{\varepsilon}=r \dot{q}_{\varepsilon}$, and $v_{\beta}=r \dot{q}_{\beta} \cos q_{\varepsilon}$, one obtains

$$
\begin{aligned}
\dot{v}_{\varepsilon} & =\dot{r} \dot{q}_{\varepsilon}-2 \dot{r} \dot{q}_{\varepsilon}-r \dot{q}_{\beta}^{2} \sin q_{\varepsilon} \cos q_{\varepsilon}+a_{t \varepsilon}-a_{m 4 \varepsilon} \\
& =-\frac{v_{r} v_{\varepsilon}}{r}-\frac{v_{\beta}^{2} \tan q_{\varepsilon}}{r}-a_{m 4 \varepsilon}+a_{t \varepsilon}, \\
\dot{v}_{\beta} & =\dot{r} \dot{q}_{\beta} \cos q_{\varepsilon}+r \ddot{q}_{\beta} \cos q_{\varepsilon}-r \dot{q}_{\varepsilon} \dot{q}_{\beta} \sin q_{\varepsilon} \\
& =-\frac{v_{r} v_{\beta}}{r}+\frac{v_{\varepsilon} v_{\beta} \tan q_{\varepsilon}}{r}+a_{m 4 \beta}-a_{t \beta},
\end{aligned}
$$

Considering the system in Equation (5) and Equation (7), one can design the following TVGESO to estimate acceleration $a_{t \varepsilon}$ of the target:

$$
\left\{\begin{array}{l}
e_{z 21}=v_{z 21}-v_{\varepsilon}, e_{z 22}=v_{z 22}-a_{t \varepsilon} \\
\dot{v}_{z 21}=v_{z 22}-\lambda_{z 21} e_{z 21}+\bar{g}_{z 2} \\
\dot{v}_{z 22}=-\lambda_{z 22} e_{z 21}
\end{array}\right.
$$

where $\bar{g}_{z 2}=-\left(v_{r} v_{\varepsilon} / r\right)-\left(v_{\beta}^{2} \tan q_{\varepsilon} / r\right)-\left(q S C_{y}^{\alpha} / m\right) \alpha ; v_{z 21}$ and $v_{z 22}$, respectively, are the estimated values of $v_{\varepsilon}$ and $a_{t \varepsilon} ; e_{z 21}$ and $e_{z 22}$ are the estimated errors; and $\lambda_{z 21}$ and $\lambda_{z 22}$ are the time-varying gain coefficients designed for the state observer. They are defined as $\lambda_{z 21}=2 L(t)$ and $\lambda_{z 22}=L^{2}(t)$, respectively. Function $L(t)$ is defined as

$$
\dot{L}(t)=\left\{\begin{array}{l}
\gamma\left|e_{z 21}\right|,\left|e_{z 21}\right| \neq 0 \\
0,\left|e_{z 21}\right|=0 \\
L(0)=\lambda_{z 0}
\end{array}\right.
$$

where $\gamma$ is the adaptive coefficient and is greater than zero. As indicated in the literature [36], appropriate values of the coefficient can ensure that the error system of the TVGESO is stable for a limited time.

Similarly, by estimating interferences $d_{z 3}$ and $d_{z 4}$ of the angle-of-attack loop and the pitch angular velocity loop, respectively, in Equation (5), one can obtain the following:

$$
\begin{aligned}
& \left\{\begin{array}{l}
e_{z 31}=v_{z 31}-x_{z 3}, e_{z 32}=v_{z 32}-d_{z 3}, \\
\dot{v}_{z 31}=v_{z 32}-\lambda_{z 31} e_{z 31}+\bar{g}_{z 3}, \\
\dot{v}_{z 32}=-\lambda_{z 32} e_{z 31},
\end{array}\right. \\
& \left\{\begin{array}{l}
e_{z 41}=v_{z 41}-x_{z 4}, e_{z 42}=v_{z 42}-d_{z 4}, \\
\dot{v}_{z 41}=v_{z 42}-\lambda_{z 41} e_{z 41}+\bar{g}_{z 4}, \\
\dot{v}_{z 42}=-\lambda_{z 42} e_{z 41},
\end{array}\right.
\end{aligned}
$$

where $\bar{g}_{z 3}=f_{z 3} x_{z 3}+x_{z 4}$ and $\bar{g}_{z 4}=f_{z 4} x_{z 3}+f_{z 5} x_{z 4}+g_{z 3} u_{z}$, the interferences $d_{z 3}$ and $d_{z 4}$ are estimated as $v_{z 32}$ and $v_{z 42}$, respectively, and the estimation errors for them are denoted by $e_{z 32}$ and $e_{z 42}$, respectively.

According to Equations (9)-(12), the interferences $a_{t \beta}$, $d_{y 3}$, and $d_{y 4}$ in the system in Equation (6) have estimated values of $v_{y 22}, v_{y 32}$, and $v_{y 42}$, respectively, with the estimation errors of $e_{y 22}, e_{y 32}$, and $e_{y 42}$, respectively.

\section{Design of the Dynamic Surface Sliding-Mode Control Law Based on Asymmetric Barrier Lyapunov Function}

Let us define $\zeta$ as an open region containing the origin and the barrier Lyapunov function $V(x)$ as a scalar function defined in $\zeta$ for the system $\dot{x}=f(x, t)$. It also has the following characteristics: (1) smooth and positive definite, (2) has a first-order continuous partial derivative at each point in $\zeta,(3)$ tends to infinity when $x$ approaches the edge of $\zeta$, and (4) satisfies the expression $V(x(t)) \leq b$ for $\forall t \geq 0$ if $x(0) \in \zeta$, where $b>0$.

Assumption 2. For any $t>0$, there exist constants $\underline{K}_{c i}$ and $\bar{K}_{c i}(i=0,1,2)$ that satisfy $\underline{k}_{c 1} \geq \underline{K}_{c 0}$ and $\bar{k}_{c 1} \leq \bar{K}_{c 0}$, with their derivatives satisfying $\left|\underline{k}_{c i}^{(i)}(t)\right| \geq \underline{K}_{c i}, \quad\left|\bar{k}_{c i}^{(i)}(t)\right| \leq \bar{K}_{c i}, \quad i=1,2$, and $\forall t \geq 0$.

Assumption 3. For any $\underline{k}_{c 1}$ 和 $\bar{k}_{c 1}$, there exist functions $\underline{Y}_{0}(t)$ and $\bar{Y}_{0}(t)$ as well as positive constants $Y_{1}$ and $Y_{2}$ satisfying $\underline{Y}_{0}(t)>\underline{k}_{c 1}(t)$ and $\bar{k}_{c 1}(t)<\bar{Y}_{0}(t)$, such that they make the system track command $x_{2 d}=\operatorname{diag}\left\{x_{z 2 d}, x_{y 2 d}\right\}$, and its time derivative satisfies $\underline{k}_{c 1}(t) \leq x_{2 d}(t) \leq \bar{k}_{c 1}(t)$ and $\left|\dot{x}_{2 d}(t)\right| \leq Y_{1}$ as well as $\left|\ddot{x}_{2 d}(t)\right| \leq Y_{2}$ for $\forall t \geq 0$. There exists a continuous set satisfying $M_{x_{2 d}}=\left\{x_{2 d} \in \mathbb{R}: x_{d}^{2}+\dot{x}_{d}^{2}+\ddot{x}_{d}^{2} \leq \phi_{x_{2 d}}\right\} \subset \mathbb{R}^{3}$.

Given that the IGC model of interceptor missile is a mismatching and uncertain system, to enable the guidance and control system to accurately pursue the target, and not violating the constraints on the control system, the control law allows using a dynamic surface sliding mode algorithm based on the asymmetric barrier Lyapunov function. It can enable the control system to pursue the target highly precisely, meanwhile ensuring that the closed-loop system is consistent and ultimately bounded, and the tracking error converges to a small set. 
4.1. Design of Control Law for Pitch Channel. For the system in Equation (5), define $x_{z 2 d}$ as the system's track command signal.

(1) Define the first dynamic error surface:

$$
s_{z 2}=x_{z 2}-x_{z 2 d}
$$

Taking the derivative of $s_{z 2}$, one obtains the error dynamic equation:

$$
\dot{s}_{z 2}=f_{z 2} x_{z 2}-x_{y 2}^{2} \sin x_{z 1} \cos x_{z 1}+\frac{a_{t \varepsilon}}{r}-\dot{x}_{z 2 d}+g_{z 2} x_{z 3} .
$$

Because the backstepping method does not have a perfect solution to the expansion of items and the problems caused by the expansion of items in the derivation process of the virtual control, this shortcoming is particularly prominent in the higher-order system. By using the dynamic surface control method and using the first-order filter to calculate the derivative of the virtual control, the expansion of the differential items can be eliminated and the controller and parameters can be designed simply [37]. Introduce virtual control variables $x_{z 3}^{*}$ and $\bar{x}_{z 3}^{*}$. To avoid complicated calculations of the expansion of the number of items during the derivation of the virtual control variables, the virtual control variable $x_{z 3}^{*}$ before filtering is passed through a first-order low-pass filter to become the virtual control variable $\bar{x}_{z 3}^{*}$ :

$$
\tau_{z 3} \dot{\bar{x}}_{z 3}^{*}+\bar{x}_{z 3}^{*}=x_{z 3}^{*}, \bar{x}_{z 3}^{*}(0)=x_{z 3}^{*}(0)
$$

In the above expression, $\tau_{z 3}$ is the filter's time constant, $\tau_{z 3}>0$, and the filtering error is defined as $y_{z 3}=\bar{x}_{z 3}^{*}-x_{z 3}^{*}$.

Considering boundary layer errors of the dynamic surface, one can construct the following asymmetric barrier Lyapunov function:

$$
\begin{aligned}
V_{z 1}= & \frac{1-p_{1}\left(s_{z 2}\right)}{2} \log \frac{k_{a 11}^{2}}{k_{a 11}^{2}-s_{z 2}^{2}} \\
& +\frac{p_{1}\left(s_{z 2}\right)}{2} \log \frac{k_{b 11}^{2}}{k_{b 11}^{2}-s_{z 2}^{2}}+\frac{1}{2} y_{z 3}^{2},
\end{aligned}
$$

where

$$
p_{1}\left(s_{z 2}\right)=\left\{\begin{array}{cc}
1 & 0<s_{z 2} \\
0 & s_{z 2} \leq 0
\end{array}\right.
$$

$k_{a 11}=x_{z 2 d}-\underline{k}_{c 1}, k_{b 11}=\bar{k}_{c 1}-x_{z 2 d}, \log (\bullet)$ represents a natural logarithm, and $k_{c 1}$ and $\bar{k}_{c 1}$ represent output constraints.

Given the independence characteristic of the output constraints $\underline{k}_{c 1}$ and $\bar{k}_{c 1}$, the tracking error constraints $k_{a 11}$ and $k_{b 11}$ can be designed independently. When constraints $k_{a 11}$ and $k_{b 11}$ are constant, and $k_{a 11} \neq k_{b 11}$, the output constrained control can be extended to a static asymmetric constraint, whereas the output constraint becomes a symmetric constraint when $k_{a 11}=k_{b 11}$. This means that the initial output can be changed depending on the setting of the constraint. It is evident that the asymmetric barrier Lyapunov function relaxes the constraint on the initial condition of the output.

As shown by Equation (16), the expression $V_{z 1} \geq 0$ simplifies to $V_{z 1}=0$ if and only if $s_{z 2}=0$ and $\bar{x}_{z 3}^{*}(0)=x_{z 3}^{*}(0)$ simultaneously. Therefore, $V_{z 1}$ is a positive-definite function in the range $-k_{a 11}<s_{z 2}<k_{b 11}$. Moreover, given $\lim _{s_{z 2} \rightarrow 0^{+}}\left(d V_{z 1} /\right.$ $\left.d s_{z 2}\right)=\lim _{s_{z 2} \rightarrow 0^{-}} d V_{z 1} / d s_{z 2}=0, V_{z 1}$ is a piecewise continuously differentiable function in the ranges $s_{z 2} \in\left(-k_{a 11}, 0\right]$ and $s_{z 2}$ $\in\left(0, k_{b 11}\right)$. Therefore, $V_{z 1}$ is a valid Lyapunov function that can ensure that the system's output error is constrained in the ranges $s_{z 2} \in\left(-k_{a 11}, 0\right]$ and $s_{z 2} \in\left(0, k_{b 11}\right)$.

Substituting the estimated values of TVGESO in Equation (9), one can design the virtual control variable for the first dynamic surface as shown in Equation (18):

$$
\begin{aligned}
x_{z 3}^{*}= & -g_{z 2}^{-1}\left(f_{z 2} x_{z 2}-x_{y 2}^{2} \sin x_{z 1} \cos x_{z 1}+\frac{v_{z 22}}{r}-\dot{x}_{z 2 d}\right) \\
& -\left(k_{z 2}+\bar{k}_{z 2}\right) s_{z 2},
\end{aligned}
$$

where $k_{z 2}>0, g_{z 2}>0$, and $r>0$, and the control coefficient $\bar{k}_{z 2}$ is defined as

$$
\bar{k}_{z 2}=\sqrt{\left(1-p_{1}\right) k_{a 11}^{2}+p_{1} k_{b 11}^{2}} .
$$

According to Equation (15), the derivative of the virtual control variable for the error surface after filtering is

$$
\dot{\bar{x}}_{z 3}^{*}=-\tau_{z 3}^{-1}\left(\bar{x}_{z 3}^{*}-x_{z 3}^{*}\right) \text {. }
$$

(2) Define the second dynamic error surface as

$$
s_{z 3}=x_{z 3}-\bar{x}_{z 3}^{*}
$$

By taking the derivation of $s_{z 3}$, one can obtain the equation for the dynamic error as follows:

$$
\dot{s}_{z 3}=\dot{x}_{z 3}-\dot{\bar{x}}_{z 3}^{*}=f_{z 3} x_{z 3}+x_{z 4}+d_{z 3}-\dot{\bar{x}}_{z 3}^{*} .
$$

Introduce two virtual control variables $x_{z 4}^{*}$ and $\bar{x}_{z 4}^{*}$. By passing the virtual control variable $x_{z 4}^{*}$ through a first-order low-pass filter, one obtains the virtual control variable $\bar{x}_{z 4}^{*}$ :

$$
\tau_{z 4} \dot{\bar{x}}_{z 4}^{*}+\bar{x}_{z 4}^{*}=x_{z 4}^{*}, \bar{x}_{z 4}^{*}(0)=x_{z 4}^{*}(0) .
$$

In Equation (23), $\tau_{z 4}$ denotes the filter time constant, $\tau_{z 4}>0$, and the filtering error is defined as $y_{z 4}=\bar{x}_{z 4}^{*}-x_{z 4}^{*}$.

Construct the following Lyapunov function:

$$
V_{z 2}=\frac{1}{2} s_{z 3}^{2}+\frac{1}{2} y_{z 4}^{2}
$$


By substituting the estimated values of TVGESO into Equation (11), one can design the virtual control variable for the second dynamic surface as shown in Equation (25):

$$
x_{z 4}^{*}=-f_{z 3} x_{z 3}-v_{z 32}+\dot{\bar{x}}_{z 3}^{*}-k_{z 3} s_{z 3}-\mu g_{z 2} s_{z 2},
$$

where $k_{z 3}>0$ and $\mu=\left(1-p_{1} / k_{a 11}^{2}-s_{z 2}^{2}\right)+\left(p_{1} / k_{b 11}^{2}-s_{z 2}^{2}\right)$.

According to Equation (23), the derivative of the virtual control variable for the error surface after filtering is

$$
\dot{\bar{x}}_{z 4}^{*}=-\tau_{z 4}^{-1}\left(\bar{x}_{z 4}^{*}-x_{z 4}^{*}\right)
$$

(3) Define the third dynamic error surface:

$$
s_{z 4}=x_{z 4}-\bar{x}_{z 4}^{*}
$$

By taking the derivative of $s_{z 4}$, one can obtain the equation of error dynamic as follows:

$$
\dot{s}_{z 4}=\dot{x}_{z 4}-\dot{\bar{x}}_{z 4}^{*}=f_{z 4} x_{z 3}+f_{z 5} x_{4}+g_{z 3} u_{z}+d_{z 4}-\dot{\bar{x}}_{z 4}^{*} .
$$

Construct the following Lyapunov function:

$$
V_{z 3}=\frac{1}{2} s_{z 4}^{2}
$$

By substituting $v_{z 42}$ with the estimated values of TVGESO in Equation (12), one can design the dynamic sliding-mode control law of the interceptor missile as shown in Equation (30):

$$
\begin{aligned}
u_{z}= & g_{z 3}^{-1}\left[-f_{z 4} x_{z 3}-f_{z 5} x_{z 4}-v_{z 42}\right. \\
& \left.+\dot{\bar{x}}_{z 4}^{*}-k_{z 4} s_{z 4}-k_{z 5}\left|s_{z 4}\right|^{\partial_{z}} \operatorname{sgn}\left(s_{z 4}\right)\right],
\end{aligned}
$$

where $k_{z 4}>0, k_{z 5}>0,0<\partial_{z}<1$, and $g_{z 3}>0$.

4.2. Stability Analysis of Control Law for Pitch Channel.

$$
\begin{gathered}
V_{1}\left(S_{1}\right) \longrightarrow \infty, \\
\gamma_{1}(\|\omega\|) \leq U(\omega) \leq \gamma_{2}(\|\omega\|),
\end{gathered}
$$

where $\gamma_{1}$ and $\gamma_{2}$ are $K_{\infty}$ functions. Suppose $V(\eta):=V_{1}\left(S_{1}\right)+$ $U(\omega)$ and $S_{1}(0) \in S_{1}$. If the inequality,

$$
\dot{V}=\frac{\partial V}{\partial \eta} h \leq-c V+v,
$$

is valid in the domain $\bar{\theta} \in N$, and $c$ and $v$ are positive constants, then $S_{1}(t)$ is still in the set for $\forall t \in[0, \infty)$.

Lemma 1 (see [20]). For any two functions $k_{a}(t)$ and $k_{b}(t)$, suppose $\mathbf{S}_{1}:=\left\{S_{1} \in \mathbb{R}:-k_{a}(t)<S_{1}<k_{b}(t)\right\} \subset \mathbb{R}$ and $N:=\mathbb{R}^{l}$ $\times \mathbf{S}_{1} \subset \mathbb{R}^{l+1}$ to be open sets. For system $\dot{\eta}=h(t, \eta)$ with $\eta:=$ $\left[\omega, S_{1}\right]^{T} \in N$ as state, function $h: \mathbb{R}_{+} \times N \rightarrow \mathbb{R}^{l+1}$ is piecewise continuous with respect to $t$, and $\mathbf{S}_{\mathbf{1}}$ satisfies the Lipschitz condition in $\mathbb{R}_{+} \times N$. If there exist two functions $U: \mathbb{R}^{l} \rightarrow \mathbb{R}_{+}$ and $V_{1}: \mathbf{S}_{1} \rightarrow \mathbb{R}_{+}$that are continuous and positively definite in their domains, respectively, the following two expressions are valid when $S_{1} \rightarrow-k_{a}(t)$ or $S_{1} \rightarrow k_{b}(t)$ :

Theorem 1. For the closed-loop system Equation (5), if the virtual control variables and the control law satisfy Equations (18), (25), and (30), then for any set $M_{z}^{0}$ to which the initial condition $y_{z}(0)$ belongs, there always exists a sufficiently large set $M_{z}:=\left\{\underline{k}_{c 1}(t)<-k_{a 11}+x_{z 2 d}(t) \leq y_{z}(t) \leq k_{b 11}+x_{z 2 d}(t)<\bar{k}_{c 1}\right.$ $(t)\}$ that can prevent the system's output constraints from being violated. Moreover, by selecting appropriate design parameters, it is possible to bound all closed-loop signals of the closed-loop system Equation (5) and make the output error converge in the neighborhood of the origin.

Proof 1. According to Equations (16), (24), and (29), one can construct a Lyapunov function that is a combination of asymmetric barrier Lyapunov functions and traditional Lyapunov functions as follows:

$$
\begin{aligned}
V_{z}= & V_{z 1}+V_{z 2}+V_{z 3}=\frac{1-p_{1}\left(s_{z 2}\right)}{2} \log \frac{k_{a 11}^{2}}{k_{a 11}^{2}-s_{z 2}^{2}} \\
& +\frac{p_{1}\left(s_{z 2}\right)}{2} \log \frac{k_{b 11}^{2}}{k_{b 11}^{2}-s_{z 2}^{2}}+\frac{1}{2} y_{z 3}^{2}+\frac{1}{2} s_{z 3}^{2}+\frac{1}{2} y_{z 4}^{2}+\frac{1}{2} s_{z 4}^{2} .
\end{aligned}
$$

By taking derivatives on both sides of the Lyapunov function in Equation (33), one obtains Equation (34):

$$
\dot{V}_{z}=\mu s_{z 2} \dot{s}_{z 2}+y_{z 3} \dot{y}_{z 3}+s_{z 3} \dot{s}_{z 3}+y_{z 4} \dot{y}_{z 4}+s_{z 4} \dot{s}_{z 4}
$$

where $\mu=\left(1-p_{1} / k_{a 11}^{2}-s_{z 2}^{2}\right)+\left(p_{1} / k_{b 11}^{2}-s_{z 2}^{2}\right)$.

Define the estimated error of the TVGESO system to satisfy Equation (35):

$$
\begin{aligned}
& \left|e_{z 22}\right|<N_{z 2} \\
& \left|e_{z 32}\right|<N_{z 3} \\
& \left|e_{z 42}\right|<N_{z 4}
\end{aligned}
$$

where $N_{z 2}, N_{z 3}$, and $N_{z 4}$ are positive constants.

It can be seen that the filtering errors are

$$
\begin{aligned}
& y_{z 3}=\bar{x}_{z 3}^{*}-x_{z 3}^{*}, \\
& y_{z 4}=\bar{x}_{z 4}^{*}-x_{z 4}^{*} .
\end{aligned}
$$

By taking derivatives of $y_{z 3}$ and $y_{z 4}$, one obtains the dynamic filtering errors:

$$
\begin{aligned}
& \dot{y}_{z 3}=-\tau_{z 3}^{-1} y_{z 3}-\dot{x}_{z 3}^{*}, \\
& \dot{y}_{z 4}=-\tau_{z 4}^{-1} y_{z 4}-\dot{x}_{z 4}^{*} .
\end{aligned}
$$


From Equations (13)-(30) and (36), one has

$$
\left\{\begin{array}{l}
x_{z 2}=s_{z 2}+x_{z 2 d}, \\
x_{z 3}=s_{z 3}+\bar{x}_{z 3}^{*}=s_{z 3}+y_{z 3}+x_{z 3}^{*}, \\
x_{z 4}=s_{z 4}+\bar{x}_{z 4}^{*}=s_{z 4}+y_{z 4}+x_{z 4}^{*}
\end{array}\right.
$$

From Equations (13)-(28) and (36)-(38), one has

$$
\begin{aligned}
\dot{s}_{z 2}= & f_{z 2} x_{z 2}-x_{y 2}^{2} \sin x_{z 1} \cos x_{z 1}+\frac{a_{t \varepsilon}}{r}-\dot{x}_{z 2 d} \\
& +g_{z 2}\left(s_{z 3}+y_{z 3}+x_{z 3}^{*}\right) \\
= & g_{z 2} s_{z 3}+g_{z 2} y_{z 3}-g_{z 2}\left(k_{z 2}+\bar{k}_{z 2}\right) s_{z 2}-\tilde{e}_{z 22},
\end{aligned}
$$

where $\tilde{e}_{z 22}=\left(v_{z 22}-a_{t \varepsilon}\right) / r$, and it is assumed that $\left|\tilde{e}_{z 22}\right|<\tilde{N}_{z 2}$, $\tilde{N}_{z 2}$ is a positive constant, and $\mu \tilde{N}_{z 2}>0$.

$$
\begin{aligned}
\dot{s}_{z 3} & =f_{z 3} x_{z 3}+s_{z 4}+y_{z 4}+x_{z 4}^{*}+d_{z 3}-\dot{x}_{z 3}^{*} \\
& =s_{z 4}+y_{z 4}-k_{z 3} s_{z 3}-e_{z 31}-\mu g_{z 2} s_{z 2}, \\
\dot{s}_{z 4} & =f_{z 4} x_{z 3}+f_{z 5} x_{z 4}+g_{z 3} u_{z}+d_{z 4}-x_{z 4}^{*} \\
& =-k_{z 4} s_{z 4}-k_{z 5}\left|s_{z 4}\right|^{\partial_{z}} \operatorname{sgn}\left(s_{z 4}\right)-e_{z 41} .
\end{aligned}
$$

According to Young's inequality and Equations (39)-(41), one has

$$
\begin{aligned}
s_{z 2} \dot{s}_{z 2}= & s_{z 2}\left[g_{z 2} s_{z 3}+g_{z 2} y_{z 3}-g_{z 2}\left(k_{z 2}+\bar{k}_{z 2}\right) s_{z 2}-\tilde{e}_{z 22}\right] \\
\leq & \left(\frac{1}{2}+\frac{1}{2} g_{z 2}-g_{z 2} k_{z 2}-g_{z 2} \bar{k}_{z 2}\right) s_{z 2}^{2}+\frac{1}{2} g_{z 2} y_{z 3}^{2} \\
& +\frac{1}{2} \tilde{N}_{z 2}^{2}+g_{z 2} s_{z 2} s_{z 3}, \\
s_{z 3} \dot{s}_{z 3}= & s_{z 3}\left(s_{z 4}+y_{z 4}-k_{z 3} s_{z 3}-e_{z 31}-\mu g_{z 2} s_{z 2}\right) \leq\left(\frac{3}{2}-k_{z 3}\right) s_{z 3}^{2} \\
& +\frac{1}{2} s_{z 4}^{2}+\frac{1}{2} y_{z 4}^{2}+\frac{1}{2} N_{z 3}^{2}-\mu g_{z 2} s_{z 2} s_{z 3}, \\
s_{z 4} \dot{s}_{z 4} & =s_{z 4}\left(-k_{z 4} s_{z 4}-k_{z 5}\left|s_{z 4}\right|^{\partial_{z}} \operatorname{sgn}\left(s_{z 4}\right)-e_{z 41}\right) \\
\leq & \left(\frac{1}{2}-k_{z 4}+\frac{3}{2} k_{z 5}\right) s_{z 4}^{2}+\frac{1}{2} N_{z 4}^{2}+\frac{1}{2} k_{z 5} .
\end{aligned}
$$

It is clear that the variables in the system model and their derivatives are all bounded. If there exist continuous functions $\tilde{z}_{z 3}$ and $\tilde{z}_{z 4}$ that satisfy $\tilde{z}_{z 3}>0$ and $\tilde{z}_{z 4}>0$, the variables $\dot{x}_{z 3}^{*}$ and $\dot{x}_{z 4}^{*}$ satisfy

$$
\begin{aligned}
& \left|\dot{x}_{z 3}^{*}\right| \leq \tilde{z}_{z 3}, \\
& \left|\dot{x}_{z 4}^{*}\right| \leq \tilde{z}_{z 4} .
\end{aligned}
$$

According to Young's inequality and Equations (36)-(37) and (45), one has

$$
\begin{gathered}
y_{z 3} \dot{y}_{z 3}=y_{z 3}\left(-\tau_{z 3}^{-1} y_{z 3}-\dot{x}_{z 3}^{*}\right) \leq-\tau_{z 3}^{-1} y_{z 3}^{2}+\frac{1}{2}\left(y_{z 3}^{2}+\tilde{z}_{z 3}^{2}\right), \\
y_{z 4} \dot{y}_{z 4}=y_{z 4}\left(-\tau_{z 4}^{-1} y_{z 4}-\dot{x}_{z 4}^{*}\right) \leq-\tau_{z 4}^{-1} y_{z 4}^{2}+\frac{1}{2}\left(y_{z 4}^{2}+\tilde{z}_{z 4}^{2}\right) .
\end{gathered}
$$

By substituting Equations (42)-(47) into Equation (34) and arranging the terms, one has

$$
\begin{aligned}
\dot{V}_{z}= & \mu s_{z 2} \dot{s}_{z 2}+y_{z 3} \dot{y}_{z 3}+s_{z 3} \dot{s}_{z 3}+y_{z 4} \dot{y}_{z 4}+s_{z 4} \dot{s}_{z 4} \\
\leq & -\mu\left(g_{z 2} k_{z 2}+g_{z 2} \bar{k}_{z 2}-\frac{1}{2} g_{z 2}-\frac{1}{2}\right) s_{z 2}^{2} \\
& -\left(k_{z 3}-\frac{3}{2}-\frac{1}{2} \mu\right) s_{z 3}^{2}-\left(k_{z 4}-1-\frac{3}{2} k_{z 5}\right) s_{z 4}^{2} \\
& -\left(\tau_{z 3}^{-1}-\frac{1}{2}-\frac{1}{2} \mu g_{z 2}\right) y_{z 3}^{2}-\left(\tau_{z 4}^{-1}-1\right) y_{z 4}^{2}+\frac{1}{2} \mu \tilde{N}_{z 2}^{2} \\
& +\frac{1}{2} N_{z 3}^{2}+\frac{1}{2} N_{z 4}^{2}+\frac{1}{2} \tilde{z}_{z 3}^{2}+\frac{1}{2} \tilde{z}_{z 4}^{2}+\frac{1}{2} k_{z 5} .
\end{aligned}
$$

For convenience of description, define the following parameters:

$$
\left\{\begin{array}{l}
K_{z 2}=g_{z 2} k_{z 2}+g_{z 2} \bar{k}_{z 2}-\frac{1}{2} g_{z 2}-\frac{1}{2}, \\
K_{z 3}=k_{z 3}-\frac{3}{2}-\frac{1}{2} \mu \\
K_{z 4}=k_{z 4}-1-\frac{3}{2} k_{z 5}, \\
\sigma_{z 3}=\tau_{z 3}^{-1}-\frac{1}{2}-\frac{1}{2} \mu g_{z 2} \\
\sigma_{z 4}=\tau_{z 4}^{-1}-1, \\
\tilde{A}=\frac{1}{2} \mu \tilde{N}_{z 2}^{2}+\frac{1}{2} N_{z 3}^{2}+\frac{1}{2} N_{z 4}^{2}+\frac{1}{2} \tilde{z}_{z 3}^{2}+\frac{1}{2} \tilde{z}_{z 4}^{2}+\frac{1}{2} k_{z 5} .
\end{array}\right.
$$

Then, Equation (48) can be rewritten as

$\dot{V}_{z} \leq-\mu K_{z 2} s_{z 2}^{2}-K_{z 3} s_{z 3}^{2}-K_{z 4} s_{4}^{2}-\sigma_{z 3} y_{z 3}^{2}-\sigma_{z 4} y_{z 4}^{2}+\tilde{A}$.

In the set $-k_{a 11}(t)<s_{z 2}<k_{b 11}(t)$, one has

$$
\begin{aligned}
& -\left(\frac{1-p_{1}}{k_{a 11}^{2}-s_{z 2}^{2}}+\frac{p_{1}}{k_{b 11}^{2}-s_{z 2}^{2}}\right) s_{z 2}^{2} \\
& \quad \leq-\left[\left(1-p_{1}\right) \log \frac{k_{a 11}^{2}}{k_{a 11}^{2}-s_{z 2}^{2}}+p_{1} \log \frac{k_{b 11}^{2}}{k_{b 11}^{2}-s_{z 2}^{2}}\right] .
\end{aligned}
$$


Then, Equation (50) can be further rewritten as

$$
\begin{aligned}
\dot{V}_{z} \leq & -\left[\left(1-p_{1}\right) \log \frac{k_{a 11}^{2}}{k_{a 11}^{2}-s_{z 2}^{2}}+p_{1} \log \frac{k_{b 11}^{2}}{k_{b 11}^{2}-s_{z 2}^{2}}\right] K_{z 2} \\
& -K_{z 3} s_{z 3}^{2}-K_{z 4} s_{z 4}^{2}-\sigma_{z 3} y_{z 3}^{2}-\sigma_{z 4} y_{z 4}^{2}+\tilde{A} .
\end{aligned}
$$

Define positive-definite matrix $Q$ as

$$
Q=\left(\begin{array}{ccc}
K_{z 2} & 0 & 0 \\
0 & K_{z 3} & 0 \\
0 & 0 & K_{z 4}
\end{array}\right)
$$

By selecting $\kappa=\min \left[2 \lambda_{\min }(Q), 2 \sigma_{z 3}, 2 \sigma_{z 4}\right]$, where $\kappa$ is a positive constant and $\lambda_{\text {min }}(Q)$ denotes the minimum eigenvalue of matrix $Q$, one has

$$
\dot{V}_{z} \leq-\kappa V_{z}+\tilde{A}
$$

Define two sets $M_{z}^{0}:=\left\{y_{z} \in \mathbb{R}: \underline{k}_{c 0} \leq y_{z}(0) \leq \bar{k}_{c 0}\right\} \subset \mathbb{R}$ and $M_{z}:=\left\{y_{z} \in \mathbb{R}: \underline{k}_{c 1} \leq y_{z}(t) \leq \bar{k}_{c 1}\right\} \subset \mathbb{R}$ that satisfy $\bar{k}_{c 1}(t)$ $>\bar{k}_{c 0}+\bar{Y}_{0}+\left|x_{z 2 d}(0)\right| \subset \mathbb{R}$ and $\underline{k}_{c 1}(t)<\underline{k}_{c 0}+\underline{Y}_{0}+\left|x_{z 2 d}(0)\right|$ $\subset \mathbb{R}$. From $s_{z 2}(0)=y_{z}(0)-x_{z 2 d}(0)$, one has

$$
-k_{a 11} \leq s_{z 2}(0) \leq k_{b 11} \text {. }
$$

Therefore, set $M_{z}$ is an invariant set. According to Lemma 1, it can be seen that given $\dot{V}_{z} \leq-\kappa V_{z}+\tilde{A}$, the following expression is valid for $\forall t \geq 0$ :

$$
-k_{a 11} \leq s_{z 2}(t) \leq k_{b 11} \text {. }
$$

According to Equations (13) and (56), one has the following expression for $\forall t \geq 0$ :

$$
\underline{k}_{c 1}(t)<-k_{a 11}+x_{z 2 d}(t) \leq y_{z}(t) \leq k_{b 11}+x_{z 2 d}(t)<\bar{k}_{c 1}(t) .
$$

Therefore, it can be concluded that for any initial compact set $M_{z}^{0}$ defined by $y_{z}(0) \in M_{z}^{0}$, there is always a sufficiently large compact set $M_{z}$ to make $y_{z} \in M_{z}$ for $\forall t \geq 0$.

Define parameters according to Equation (48) to satisfy the following rules:

$$
\left\{\begin{array}{l}
k_{z 2} \geq \frac{1}{2} g_{z 2}^{-1}+\frac{1}{2}-\bar{k}_{z 2}+\frac{1}{2} \kappa, \quad k_{z 3} \geq \frac{3}{2}+\frac{1}{2} \mu+\frac{1}{2} \kappa, \quad k_{z 4} \geq \frac{3}{2} k_{z 5}+1+\frac{1}{2} \kappa, \\
\tau_{z 3}^{-1} \geq \frac{1}{2}+\frac{1}{2} \mu g_{z 2}+\frac{1}{2} \kappa, \quad \tau_{z 4}^{-1} \geq 1+\frac{1}{2} \kappa .
\end{array}\right.
$$

Multiply both sides of Equation (54) by $e^{\kappa t}$ and arrange the terms to obtain the following expression:

$$
V_{z}(t) \leq \frac{\left\{\left[\kappa V_{z}(0)-\tilde{A}\right] e^{-\kappa t}+\tilde{A}\right\}}{\kappa}
$$

Therefore, it is clear that by designing parameters $k_{z 2}, k_{z 3}$, $k_{z 4}$, and $k_{z 5}$ and parameters $\tau_{z 3}$ and $\tau_{z 4}$, it is possible to ensure that all closed-loop signals of the system are bounded. Opting to increase $k_{z 2}, k_{z 3}, k_{z 4}$, and $k_{z 5}$ and decrease $\tau_{z 3}$ and $\tau_{z 4}$ can ensure that $\kappa$ is sufficiently large to make the filtering errors and the error surface small enough to control accuracy. Q.E.D.

Remark 1. Theoretically, larger values of the designed parameters $k_{z 2}, k_{z 3}, k_{z 4}$, and $k_{z 5}$; smaller ones of $\tau_{z 3}$ and $\tau_{z 4}$; the final boundary of the resulting error surfaces $s_{z 2}$, $s_{z 3}$, and $s_{z 4}$; and filtering errors $y_{z 3}$ and $y_{z 4}$ indicate an increase in the precision of control. However, in practice, very large values of $k_{z 2}, k_{z 3}, k_{z 4}$, and $k_{z 5}$, and very small ones of $\tau_{z 3}$ and $\tau_{z 4}$ can easily lead to input saturation in interceptor missile control systems, which triggers the saturation nonlinearity of the system such that the required overloads of the interceptor missile are beyond the available capacity, resulting in a reduction in the system's control performance. Moreover, given the physical limitations of the low-pass filter, $\tau_{z 3}$ and $\tau_{z 4}$ should not be chosen to be arbitrarily small. Therefore, the parameters of the control algorithm should be properly selected in light of practical considerations.

4.3. Design of Control Law for Yaw Channel. Based on the design of the control law for the pitch channel, one can substitute $v_{y 22}, v_{y 32}$, and $v_{y 42}$-the estimated interferences of the TVGESO - into the system of Equation (6) and define $x_{y 2 d}$ as the system's track command sign for the following form of control law for the yaw channel: 


$$
\left\{\begin{array}{l}
s_{y 2}=x_{y 2}-x_{y 2 d}, \\
\tau_{y 3} \dot{\bar{x}}_{y 3}^{*}+\bar{x}_{y 3}^{*}=x_{y 3}^{*}, \bar{x}_{y 3}^{*}(0)=x_{y 3}^{*}(0), \\
x_{y 3}^{*}=-g_{y 2}^{-1}\left(f_{y 2} x_{y 2}+2 x_{z 2} x_{y 2} \tan x_{z 1}-\frac{v_{y 22}}{r \cos x_{z 1}}-\dot{x}_{y 2 d}\right)-\left(k_{y 2}+\bar{k}_{y 2}\right) s_{y 2}, \\
s_{y 3}=x_{y 3}-\bar{x}_{y 3}^{*}, \\
\tau_{y 4} \dot{\bar{x}}_{y 4}^{*}+\bar{x}_{y 4}^{*}=x_{y 4}^{*}, \bar{x}_{y 4}^{*}(0)=x_{y 4}^{*}(0), \\
x_{y 4}^{*}=-f_{y 3} x_{y 3}-v_{y 32}+\dot{\bar{x}}_{y 3}^{*}-k_{y 3} s_{y 3}-\tilde{\mu} g_{y 2} s_{y 2}, \\
s_{y 4}=x_{y 4}-\bar{x}_{y 4}^{*}, \\
u_{y}=g_{y 3}^{-1}\left[-f_{y 4} x_{y 3}-f_{y 5} x_{y 4}-v_{y 42}+\dot{\bar{x}}_{y 4}^{*}-k_{y 4} s_{y 4}-k_{y 5}\left|s_{y 4}\right|^{\partial_{y}} \operatorname{sgn}\left(s_{y 4}\right)\right],
\end{array}\right.
$$

where $\bar{k}_{y 2}=\sqrt{\left(1-p_{2}\right) k_{a 12}^{2}+p_{2} k_{b 12}^{2}}$,

$$
p_{2}\left(s_{y 2}\right)= \begin{cases}1 & 0<s_{y 2} \\ 0 & s_{y 2} \leq 0\end{cases}
$$

$k_{a 12}=x_{y 2 d}-\underline{k}_{c 1}, \quad k_{b 12}=\bar{k}_{c 1}-x_{y 2 d}, \quad \tilde{\mu}=\left(1-p_{2} / k_{a 12}^{2}-s_{y 2}^{2}\right)+$ $\left(p_{2} / k_{b 12}^{2}-s_{y 2}^{2}\right), \quad k_{y 2}>0, \quad k_{y 3}>0, k_{y 4}>0, k_{y 5}>0,0<\partial_{y}<1$, $g_{y 2}>0, g_{y 3}>0$, and $r>0$. The parameters $s_{y 2}, s_{y 3}$, and $s_{y 4}$ are error surfaces; $\tau_{y 3}$ and $\tau_{y 4}$ are filter time constants, $\tau_{y 3}>0$ and $\tau_{y 4}>0 ; x_{y 3}^{*}$ and $x_{y 4}^{*}$ are virtual control variables of the system Equation (6); and $\bar{x}_{y 3}^{*}$ and $\bar{x}_{y 4}^{*}$, respectively, are virtual control variables after filtering.

According to the stability analysis method in Equations (33)-(59), it can be proven that the closed-loop system of Equation (6) is stable and the system's control precision can be achieved by designing appropriate parameters.

\section{Dynamic Control Allocation Algorithm}

Based on Equations (30) and (60), one can obtain the control moments jointly generated by the aerodynamic rudders and the jet devices using control inputs $u_{z}$ and $u_{y}$ as the desired control moments. Therefore, it is necessary to use a dynamic control allocation technique to distribute the desired control moments to the actuators.

Define virtual control moment $u$ as

$$
u=B \delta
$$

where $u=\left[u_{z}, u_{y}\right]^{T}, \delta=\left[\delta_{z}, \delta_{s y}, \delta_{y}, \delta_{s z}\right]^{T}$,

$$
B=\left[\begin{array}{cccc}
B_{z 1} & B_{z 2} & 0 & 0 \\
0 & 0 & B_{y 1} & B_{y 2}
\end{array}\right]^{T},
$$

$B_{z 1}=q S \bar{L} m_{z}^{\delta_{z}}, B_{z 2}=\left(1+K_{z}\right) F_{s \max } L_{m}, B_{y 1}=q S \bar{L} m_{y}^{\delta_{y}}$, and $B_{y 2}=\left(1+K_{y}\right) F_{s \max } L_{m}$.
Given that actuators are subject to physical constraints, such as structural and load-related constraints, the range of deflection and speed are limited. Define the control moment as in the feasible range $\delta_{\min }(t) \leq \delta(t) \leq \delta_{\max }(t)$. To achieve stable control performance, define the rate of change of the control moment to satisfy $\dot{\delta}_{\min }(t) \leq \dot{\delta}(t) \leq \dot{\delta}_{\max }(t)$, where $\delta_{\min }$ and $\delta_{\max }$ are the minimum and maximum positional constraints of the actuators, respectively, with $\dot{\delta}_{\text {min }}$ and $\dot{\delta}_{\text {max }}$ being, respectively, the minimum and maximum speed constraints. Using $T$ to denote sampling time, one can rewrite the feasible range of the actuators as

$$
\underline{\delta}(t) \leq \delta(t) \leq \bar{\delta}(t)
$$

where $\underline{\delta}(t)=\max \left\{\delta_{\min }, \delta(t-T)+\dot{\delta}_{\min } T\right\}$ and $\bar{\delta}(t)=\min$ $\left\{\delta_{\max }, \delta(t-T)+\dot{\delta}_{\max } T\right\}$.

To achieve stable and smooth actuator trajectories to suppress the impacts of noise and interference on the controller, a dynamic control allocation algorithm is designed to solve the allocation problem between the aerodynamic rudders and the jet devices.

The dynamic control allocation problem can be expressed as the following hybrid optimization problem:

$$
\begin{array}{ll}
\min _{\delta(t)} & J_{0}+J_{1}+J_{2}, \\
\text { subject to } & u=B \delta, \quad \underline{\delta}(t) \leq \delta(t) \leq \bar{\delta}(t),
\end{array}
$$

where $\quad J_{0}=\left\|W_{0} \delta(t)\right\|^{2}, \quad J_{2}=\left\|W_{2}[\delta(t)-\delta(t-T)]\right\|^{2}+$ $\left\|W_{2}[\delta(t)-\delta(t-2 T)]\right\|^{2}, \quad J_{1}=\left\|W_{1}\left[\delta(t)-\delta_{s}(t)\right]\right\|^{2}, \quad W_{0}=$ $W_{0}^{T}>0, W_{1}=W_{1}^{T}>0$, and $W_{2}=W_{2}^{T}>0$.

$\|\cdot\|$ represents a norm defined as $\|x\|=\sqrt{x^{T} x}, \delta_{s}(t)$ denotes the desired control command of the actuators, $J_{0}$ denotes the minimum energy principle, $J_{1}$ denotes the minimum error principle for the desired control commands of the actuators, and $J_{2}$ denotes the minimum transition rate principle for the relative sampling time of the expected control commands. 
Define diagonal matrices $R_{0}, R_{1}$, and $R_{2}$ with corresponding dimensions

$$
\left\{\begin{array}{l}
R_{0}=\operatorname{diag}\left\{R_{01}, R_{02}, R_{03}, R_{04}\right\}, \\
R_{1}=\operatorname{diag}\left\{R_{11}, R_{12}, R_{13}, R_{14}\right\}, \\
R_{2}=\operatorname{diag}\left\{R_{21}, R_{22}, R_{23}, R_{24}\right\},
\end{array}\right.
$$

where

$$
\begin{aligned}
& R_{0 i}= \begin{cases}\frac{\bar{\delta}_{i}(t)}{\delta_{i}(t)}, & \delta_{i}(t)>\bar{\delta}_{i}(t) \\
1, & \underline{\delta}_{i}(t) \leq \delta_{i}(t) \leq \bar{\delta}_{i}(t) \\
\bar{\delta}_{i}(t) & \delta_{i}(t)<\underline{\delta}_{i}(t)\end{cases} \\
& R_{1 i}= \begin{cases}\frac{\bar{\delta}_{s i}(t)}{\delta_{s i}(t)}, & \delta_{s i}(t)>\bar{\delta}_{s i}(t), \\
1, & \underline{\delta}_{s i}(t) \leq \delta_{s i}(t) \leq \bar{\delta}_{s i}(t), \\
\bar{\delta}_{s i}(t) & \delta_{s i}(t)<\underline{\delta}_{s i}(t),\end{cases}
\end{aligned}
$$

and

$$
R_{2 i}= \begin{cases}\frac{\bar{\delta}_{i}(t-T)}{\delta_{i}(t-T)}, & \delta_{i}(t-T)>\bar{\delta}_{i}(t-T), \\ 1, & \underline{\delta}_{i}(t-T) \leq \delta_{i}(t-T) \leq \bar{\delta}_{i}(t-T), \\ \bar{\delta}_{i}(t-T) & \delta_{i}(t-T)<\underline{\delta}_{i}(t-T),\end{cases}
$$

with $\delta_{i}(t), \bar{\delta}_{i}(t), \underline{\delta}_{i}(t), \delta_{s i}(t), \bar{\delta}_{s i}(t), \underline{\delta}_{s i}(t), \delta_{i}(t-T), \bar{\delta}_{i}(t-T)$, and $\underline{\delta}_{i}(t-T)$ the elements of the corresponding vectors $i \in$ $\{1,2,3,4\}$.

Theorem 2. The hybrid optimization problem in Equation (65) is equivalent to

$$
\begin{array}{ll}
\min _{\delta(t)} & \bar{J}_{0}+\bar{J}_{1}+\bar{J}_{2}, \\
\text { subject to } & u=B \delta, \quad \underline{\delta}(t) \leq \delta(t) \leq \bar{\delta}(t),
\end{array}
$$

where $\bar{J}_{0}=\left\|R_{0} W_{0} \delta(t)\right\|^{2}, \quad \bar{J}_{2}=\left\|R_{2} W_{2}[\delta(t)-\delta(t-T)]\right\|^{2}+$ $\left\|W_{2}[\delta(t)-\delta(t-2 T)]\right\|^{2}$, and $\bar{J}_{1}=\left\|R_{1} W_{1}\left[\delta(t)-\delta_{s}(t)\right]\right\|^{2}$, and the general solution is given in Equation (70):

$$
\delta(t)=E \delta_{s}(t)+F_{1} \delta(t-T)+F_{2} \delta(t-2 T)+G u(t)
$$

where $\quad E=(I-G B) W^{-2} R_{1}^{2} W_{1}^{2}, \quad F_{1}=(I-G B) W^{-2} R_{2}^{2} W_{2}^{2}$, $F_{2}=(I-G B) W^{-2} W_{2}^{2}, \quad G=W^{-2} B^{T}\left(B W^{-2} B^{T}\right)^{-1}$, and $W=$ $\left(R_{0}^{2} W_{0}^{2}+R_{1}^{2} W_{1}^{2}+R_{2}^{2} W_{2}^{2}+W_{2}^{2}\right)^{1 / 2}$.
Proof 2. From Equation (69), one has

$$
\begin{aligned}
\bar{J}= & \bar{J}_{0}+\bar{J}_{1}+\bar{J}_{2}=\delta^{T}(t) R_{0}^{2} W_{0}^{2} \delta+\left[\delta(t)-\delta_{s}(t)\right]^{T} R_{1}^{2} W_{1}^{2}[\delta(t) \\
& \left.-\delta_{s}(t)\right]+[\delta(t)-\delta(t-T)]^{T} R_{2}^{2} W_{2}^{2}[\delta(t)-\delta(t-T)] \\
& +[\delta(t)-\delta(t-2 T)]^{T} W_{2}^{2}[\delta(t)-\delta(t-2 T)] \\
= & \left\|W\left[\delta(t)-\delta_{0}^{*}(t)\right]\right\|^{2},
\end{aligned}
$$

where $\delta_{0}^{*}(t)=W^{-2}\left[R_{1}^{2} W_{1}^{2} \delta_{s}(t)+R_{2}^{2} W_{2}^{2} \delta(t-T)+W_{2}^{2} \delta(t-2\right.$ $T)$ ]. Therefore, the optimization problem in Equation (69) is equivalent to

$$
\begin{aligned}
& \min _{\delta(t)}=\left\|W\left[\delta(t)-\delta_{0}^{*}(t)\right]\right\|^{2} \\
& \text { subject to } \quad u=B \delta, \quad \underline{\delta}(t) \leq \delta(t) \leq \bar{\delta}(t) .
\end{aligned}
$$

According to the weighted pseudo-inverse theorem [38], the general solution of the optimization problem in Equation (69) is

$$
\delta(t)=(I-G B) \delta_{0}^{*}(t)+G u(t)
$$

By substituting Equation (71) into Equation (73), one can find that Theorem 2 is valid. Q.E.D.

Remark 2. By including the minimum error term related to the error between the command of the given step and those one or two steps prior, the purpose is to reduce chattering in the system and smoothen the control commands. By increasing the coefficient matrices $R_{0}, R_{1}$, and $R_{2}$, one can solve the constraint problem of control moment to solve the optimization problem.

\section{Simulation Analysis}

To verify the effectiveness of the asymmetric barrier Lyapunov function-based IGC algorithm designed in this study for the interceptor missile, it was assumed that given an interceptor missile, its initial position was $(X m, Y m, Z m)=(0 \mathrm{~m}$, $0 \mathrm{~m}, 0 \mathrm{~m})$, its initial velocity was $V_{m 0}=1600 \mathrm{~m} / \mathrm{s}$, and the initial position of the target was $(X t, Y t, Z t)=(5000 \mathrm{~m}$, $6000 \mathrm{~m}, 4000 \mathrm{~m}$ ), and the initial velocity was $V_{t 0}=1200 \mathrm{~m} / \mathrm{s}$. The rudder deflection angle of the interceptor missile was limited in the range $-30^{\circ}$ to $+30^{\circ}$, the angular velocity of the rudder's deflection was limited in the range $-300^{\circ} / \mathrm{s}$ to $+300^{\circ}$ s, the maximum steady-state thrust of the jet devices was $F_{s \max }=4800 \mathrm{~N}$, and the their moment amplification factor was $K_{z}=K_{y}=0.3$. The asymmetric output constraints were $\underline{k}_{c 1}=-4$ and $\bar{k}_{c 1}=2$, and the time-varying asymmetric output constraints were $\underline{k}_{c 1}=-4+0.2 \cos (t)$ and $\bar{k}_{c 1}=2+$ $0.1 \mathrm{sin}(t)$. The aerodynamic parameters of the interceptor missile are shown in Table 1.

The method designed in this study (i.e., ABLFIGC+ DCA) was compared with the combined use of IGC and the traditional dynamic surface sliding-mode control law (i.e., IGC+DCA). Assuming that the interferences in the 
TABLe 1: Aerodynamic parameters of the interceptor missile.

\begin{tabular}{lccc}
\hline Parameter & Value & Parameter & Value \\
\hline$J_{z}$ & $247.43 \mathrm{~kg} \cdot \mathrm{m}^{2}$ & $C_{z}^{\beta}$ & 19.79 \\
$J_{y}$ & $247.43 \mathrm{~kg} \cdot \mathrm{m}^{2}$ & $m_{z}^{\alpha}$ & -15.26 \\
$m$ & $204 \mathrm{~kg}$ & $m_{y}^{\beta}$ & -15.26 \\
$\bar{L}$ & $0.22 \mathrm{~m}$ & $m_{z}^{\omega_{z}}$ & -0.16 \\
$L_{m}$ & $2 \mathrm{~m}$ & $m_{y}^{\omega_{y}}$ & -0.16 \\
$S$ & $0.0409 \mathrm{~m}^{2}$ & $m_{z}^{\delta_{z}}$ & -11.81 \\
$C_{y}^{\alpha}$ & -19.79 & $m_{y}^{\delta_{y}}$ & -11.81 \\
\hline
\end{tabular}

system were $d_{z 3}=d_{z 4}=d_{y 3}=d_{y 4}=0.02 \sin (t)$, target interception was maneuvered in the following two scenarios:

Scenario 1. The target was in uniform motion: $a_{t \varepsilon}=a_{t \beta}=0$ $\mathrm{m} / \mathrm{s}^{2}$.

Scenario 2. The target was in accelerated motion: $a_{t \varepsilon}=a_{t \beta}=$ $10 \mathrm{~m} / \mathrm{s}^{2}$.

When the asymmetric output constraints were $\underline{k}_{c 1}=-4$ and $\bar{k}_{c 1}=2$, the simulation comparison plots of Scenario 1 are shown in Figures 2-7.

Figures 2 and 3, respectively, show the curves of angular velocities of the vertical and horizontal lines of sight of the interceptor missile in Scenario 1. It is clear that a certain degree of chattering occurred during convergence when the traditional IGC algorithm was used, and the two angular velocities exhibited large jumps in the late stage of guidance. By contrast, the ABLFIGC+DCA algorithm designed in this study caused the two angular velocities to converge more quickly to steady-state values in a smoother convergence process that met the asymmetric output constraints $\underline{k}_{c 1}$ and $\bar{k}_{c 1}$, thus improving the stability of the guidance and control system and showing good robustness against external interferences.

Figures 4-7 show the curves of the attack angle, sideslip angle, and angular velocities of the pitch and yaw of the interceptor missile in Scenario 1, respectively. A certain degree of chattering appeared due to interference when the traditional IGC algorithm was used, and the above four parameters all exhibited large jumps in the late stage of guidance. By contrast, the ABLFIGC+DCA algorithm designed in this study renders the entire convergence process smoother, with the above four parameters stable and not recording large jumps in the late stage of guidance. Moreover, this algorithm showed good robustness against external interference.

The simulation comparison plots of Scenario 2 are shown in Figures 8-13.

Figures 8-13 show the simulation comparison plots of the target motion Scenario 2. From Figures 8 and 9, it is clear that the ABLFIGC+DCA algorithm smoothened convergence and was free of large jumps in the guidance process, met the asymmetric output constraints $\underline{k}_{c 1}$ and $\bar{k}_{c 1}$, and

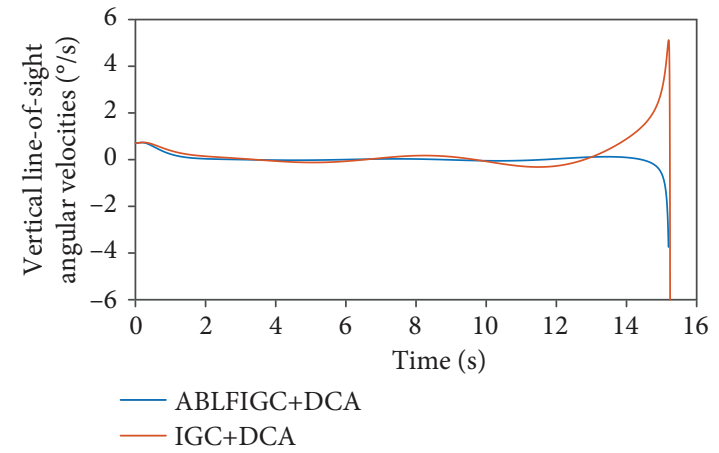

FIgURE 2: Curves of angular velocities of vertical line of sight of the interceptor missile in Scenario 1: ABLFIGC+DCA refers to a combination of the proposed asymmetric barrier Lyapunov function-based IGC algorithm and the dynamic control allocation algorithm; IGC+DCA refers to a combination of the IGC algorithm subject to the traditional dynamic surface sliding-mode control law and the dynamic control allocation algorithm.

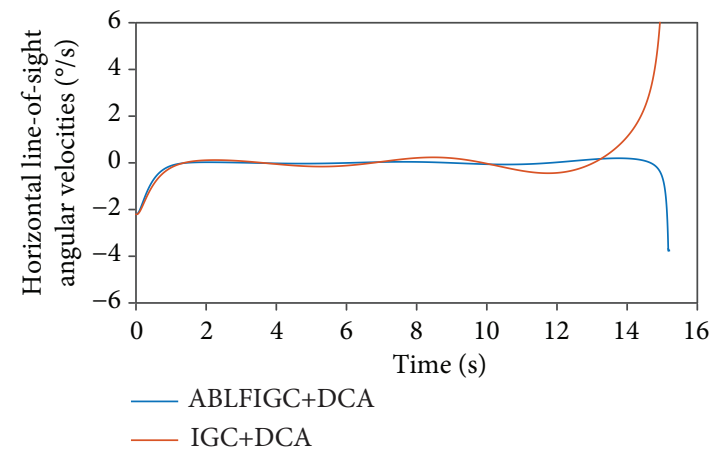

Figure 3: Curves of angular velocities of horizontal line of sight of the interceptor missile in Scenario 1: ABLFIGC+DCA refers to a combination of the proposed asymmetric barrier Lyapunov function-based IGC algorithm and the dynamic control allocation algorithm; IGC+DCA refers to a combination of the IGC algorithm subject to the traditional dynamic surface sliding-mode control law and the dynamic control allocation algorithm.

showed good robustness against external interference. As shown in Figures 10-13, the ABLFIGC+DCA algorithm ensured that the flight attitude of the interceptor missile was stable and changed smoothly.

The results of comparison of the two scenarios in terms of the target miss distance and interception time of the interceptor missile are shown in Table 2 .

As shown in Table 2, the algorithm designed in this study had a smaller miss distance and interception time than the IGC algorithm subject to the traditional dynamic slidingmode control law.

For Scenario 1, the simulation comparison plots of $\delta_{z}, \delta_{s y}$, $\delta_{y}$, and $\delta_{s z}$ of the actuators obtained by the dynamic control allocation algorithm are shown in Figures 14-17.

Figures 14-17 show the curves of the elevator deflection angle $\delta_{z}$, the rudder deflection angle rudder angle $\delta_{y}$, and the equivalent rudder deflection angles $\delta_{s z}$ and $\delta_{s y}$, respectively, all of which were obtained via the dynamic control allocation 


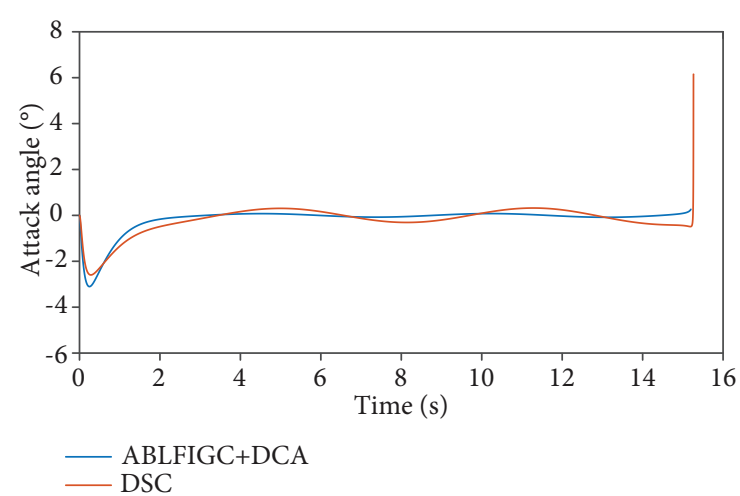

FIGURE 4: Attack angle curves of the interceptor missile in Scenario 1: ABLFIGC+DCA refers to a combination of the proposed asymmetric barrier Lyapunov function-based IGC algorithm and the dynamic control allocation algorithm; IGC+DCA refers to a combination of the IGC algorithm subject to the traditional dynamic surface sliding-mode control law and the dynamic control allocation algorithm.

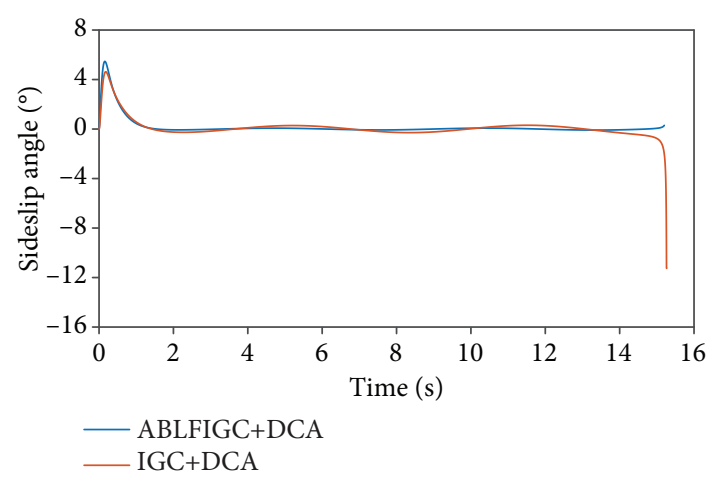

FIGURE 5: Sideslip angle curves of the interceptor missile in Scenario 1: ABLFIGC+DCA refers to a combination of the proposed asymmetric barrier Lyapunov function-based IGC algorithm and the dynamic control allocation algorithm; IGC+DCA refers to a combination of the IGC algorithm subject to the traditional dynamic surface sliding-mode control law and the dynamic control allocation algorithm.

algorithm. As shown in these figures, the ABLFIGC+DCA algorithm rendered the actuator outputs bounded and caused them to alter smoothly, had good robustness against external interferences, and did not incur a large jump in the late stage of guidance, indicating a good control allocation performance.

When the time-varying asymmetric output constraints were $\underline{k}_{c 1}=-4+0.2 \cos (t)$ and $\bar{k}_{c 1}=2+0.1 \sin (t)$, due to the attack angle, sideslip angle, and angular velocities of the pitch and yaw of the interceptor missile which are similar to the above, therefore, only the angular velocities of the vertical and horizontal lines of sight of the interceptor missile are compared. The simulation comparison plots of Scenario 1 are shown in Figures 18 and 19.

The simulation comparison plots of Scenario 2 are shown in Figures 20 and 21.

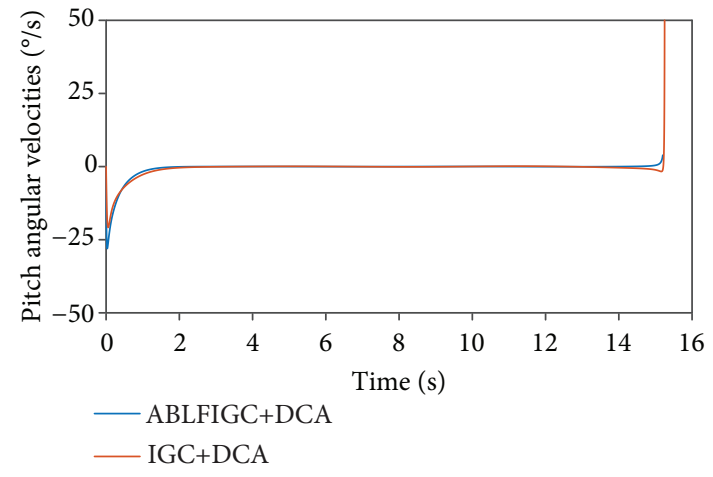

FIGURE 6: Pitch angular velocity curves of the interceptor missile in Scenario 1: ABLFIGC+DCA refers to a combination of the proposed asymmetric barrier Lyapunov function-based IGC algorithm and the dynamic control allocation algorithm; IGC+DCA refers to a combination of the IGC algorithm subject to the traditional dynamic surface sliding-mode control law and the dynamic control allocation algorithm.

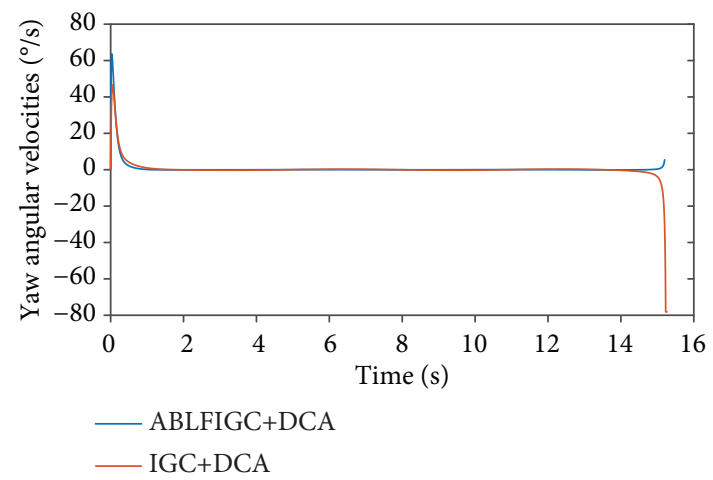

FIgURE 7: Yaw angular velocity curves of the interceptor missile in Scenario 1: ABLFIGC+DCA refers to a combination of the proposed asymmetric barrier Lyapunov function-based IGC algorithm and the dynamic control allocation algorithm; IGC+ DCA refers to a combination of the IGC algorithm subject to the traditional dynamic surface sliding-mode control law and the dynamic control allocation algorithm.

Figures 18-21, respectively, show the curves of angular velocities of the vertical and horizontal lines of sight of the interceptor missile in Scenario 1 and Scenario 2. From Figures 18 and 21, it is clear that the ABLFIGC+DCA algorithm smoothened convergence and was free of large jumps in the guidance process, met the asymmetric output constraints $\underline{k}_{c 1}=-4+0.2 \cos (t)$ and $\bar{k}_{c 1}=2+0.1 \sin (t)$, and showed good robustness against external interference.

As shown by the above simulation results, the asymmetric barrier Lyapunov function-based IGC algorithm proposed in this study for the interceptor missile, when compared with the conventional IGC algorithm, can enable the outputs to meet constraints and the system to have better control performance and anti-interference ability, thus improving the stability of the guidance and control systems for the interceptor missile. 


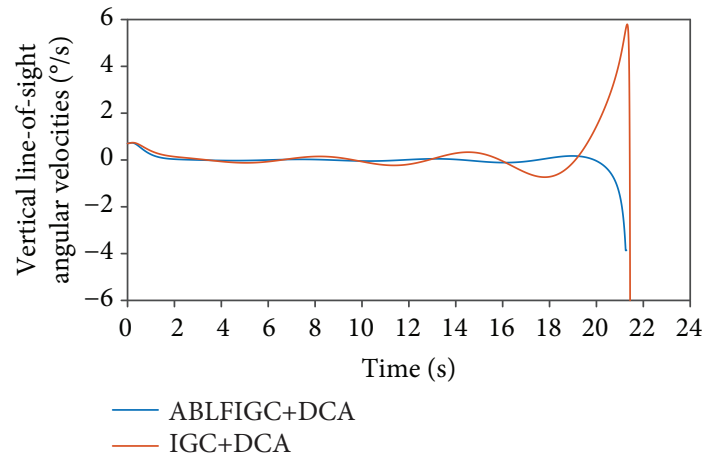

FIGURE 8: Curves of angular velocities of vertical line of sight of the interceptor missile in Scenario 2: ABLFIGC+DCA refers to a combination of the proposed asymmetric barrier Lyapunov function-based IGC algorithm and the dynamic control allocation algorithm; IGC+DCA refers to a combination of the IGC algorithm subject to the traditional dynamic surface sliding-mode control law and the dynamic control allocation algorithm.

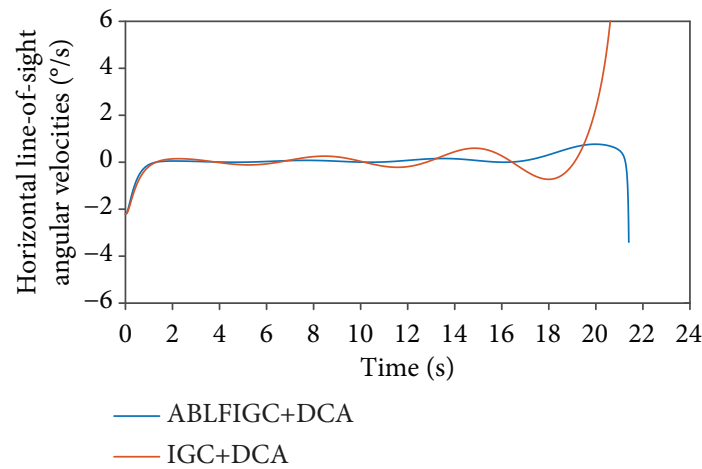

FIGURE 9: Curves of angular velocities of the horizontal line of sight of the interceptor missile in Scenario 2: ABLFIGC+DCA refers to a combination of the proposed asymmetric barrier Lyapunov function-based IGC algorithm and the dynamic control allocation algorithm; IGC+DCA refers to a combination of the IGC algorithm subject to the traditional dynamic surface sliding-mode control law and the dynamic control allocation algorithm.

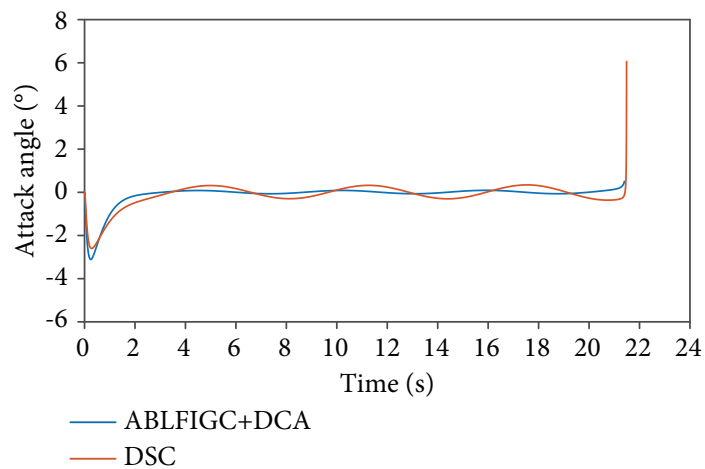

FIGURE 10: Attack angle curves of the interceptor missile in Scenario 2: ABLFIGC+DCA refers to a combination of the proposed asymmetric barrier Lyapunov function-based IGC algorithm and the dynamic control allocation algorithm; IGC+DCA refers to a combination of the IGC algorithm subject to the traditional dynamic surface sliding-mode control law and the dynamic control allocation algorithm.

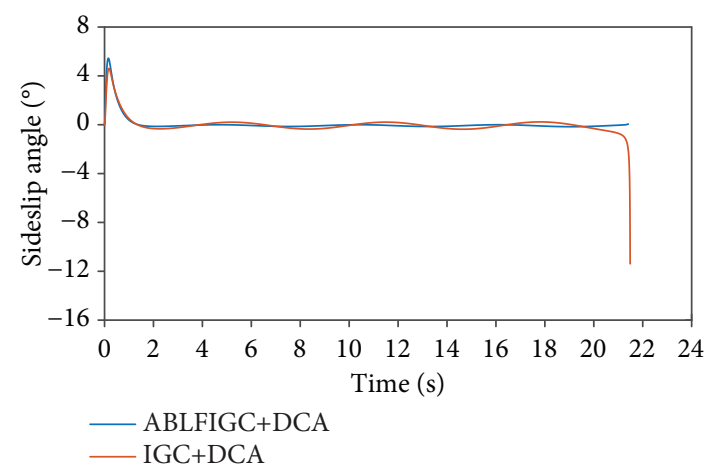

FIgURE 11: Sideslip angle curves of the interceptor missile in Scenario 2: ABLFIGC+DCA refers to a combination of the proposed asymmetric barrier Lyapunov function-based IGC algorithm and the dynamic control allocation algorithm; IGC+ DCA refers to a combination of the IGC algorithm subject to the traditional dynamic surface sliding-mode control law and the dynamic control allocation algorithm.

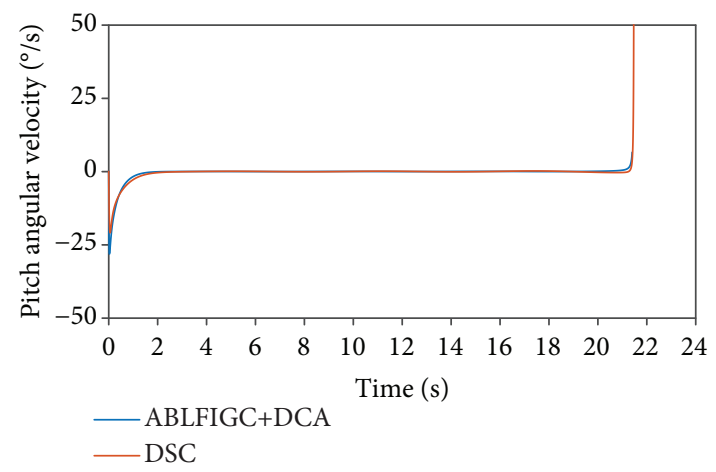

FIGURE 12: Pitch angular velocity curves of the interceptor missile in Scenario 2: ABLFIGC+DCA refers to a combination of the proposed asymmetric barrier Lyapunov function-based IGC algorithm and the dynamic control allocation algorithm; IGC+DCA refers to a combination of the IGC algorithm subject to the traditional dynamic surface sliding-mode control law and the dynamic control allocation algorithm.

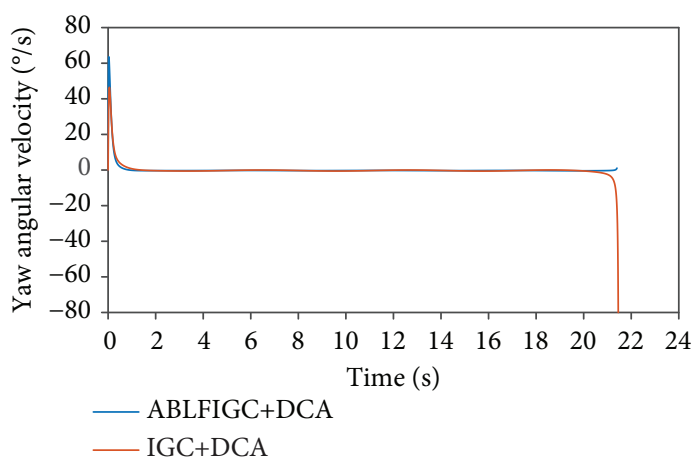

FIgURE 13: Yaw angular velocity curves of the interceptor missile in Scenario 2: ABLFIGC+DCA refers to a combination of the proposed asymmetric barrier Lyapunov function-based IGC algorithm and the dynamic control allocation algorithm; IGC+DCA refers to a combination of the IGC algorithm subject to the traditional dynamic surface sliding-mode control law and the dynamic control allocation algorithm. 
TABle 2: Comparison of simulation results between the two scenarios.

\begin{tabular}{lccc}
\hline Scenario & Control mode & $\begin{array}{c}\text { Miss } \\
\text { distance }(\mathrm{m})\end{array}$ & $\begin{array}{c}\text { Interception } \\
\text { time }(\mathrm{s})\end{array}$ \\
\hline \multirow{2}{*}{1} & ABLFIGC+DCA & 0.608 & 15.21 \\
& IGC + DCA & 0.792 & 15.26 \\
\hline \multirow{2}{*}{2} & ABLFIGC+DCA & 0.854 & 21.42 \\
& IGC + DCA & 0.992 & 21.48 \\
\hline
\end{tabular}

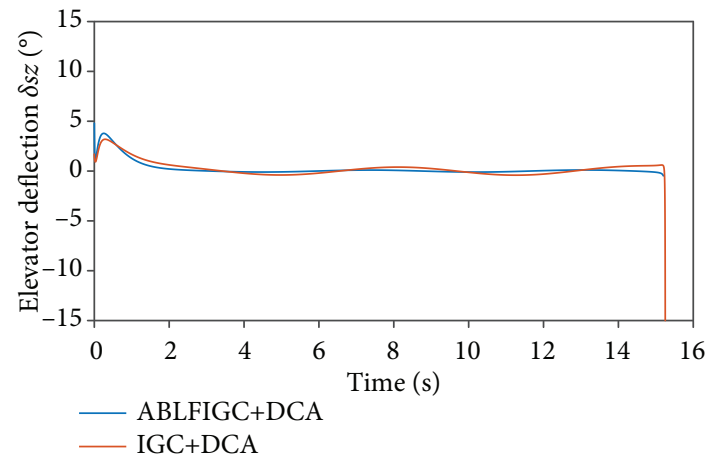

FIGURE 14: Curves of elevator deflection angle $\delta_{z}$ : ABLFIGC+DCA refers to a combination of the proposed asymmetric barrier Lyapunov function-based IGC algorithm and the dynamic control allocation algorithm; IGC+DCA refers to a combination of the IGC algorithm subject to the traditional dynamic surface slidingmode control law and the dynamic control allocation algorithm.

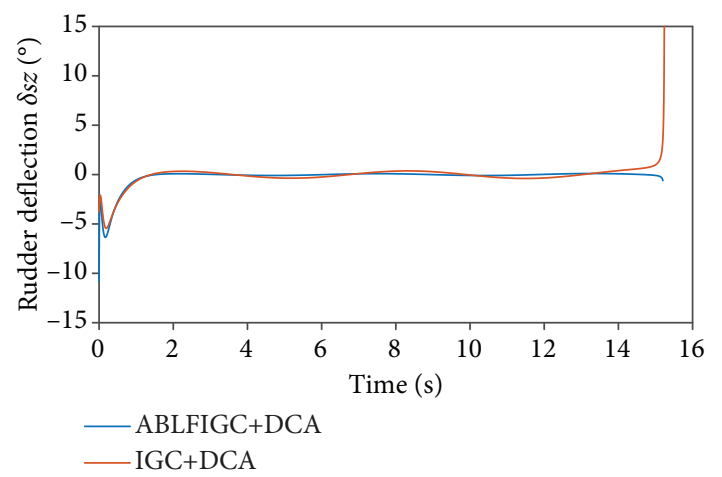

FIgURE 15: Curves of rudder deflection angle $\delta_{y}$ : ABLFIGC+DCA refers to a combination of the proposed asymmetric barrier Lyapunov function-based IGC algorithm and the dynamic control allocation algorithm; IGC+DCA refers to a combination of the IGC algorithm subject to the traditional dynamic surface slidingmode control law and the dynamic control allocation algorithm.

\section{Conclusion}

In the context of the interceptor missile which uses a directforce/aerodynamic-force control scheme, by considering the coupling relationship between the pitch and yaw channels of the interceptor missile as well as the output constraint

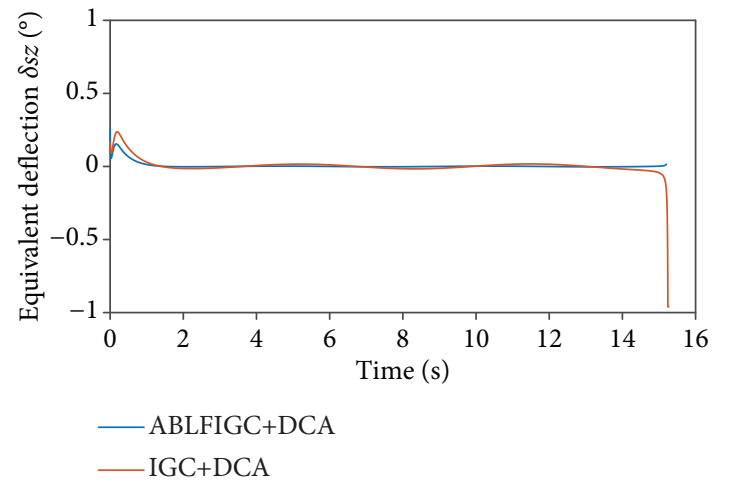

FIgURE 16: Curves of equivalent rudder deflection angle $\delta_{s z}$ : ABLFIGC+DCA refers to a combination of the proposed asymmetric barrier Lyapunov function-based IGC algorithm and the dynamic control allocation algorithm; IGC+DCA refers to a combination of the IGC algorithm subject to the traditional dynamic surface sliding-mode control law and the dynamic control allocation algorithm.

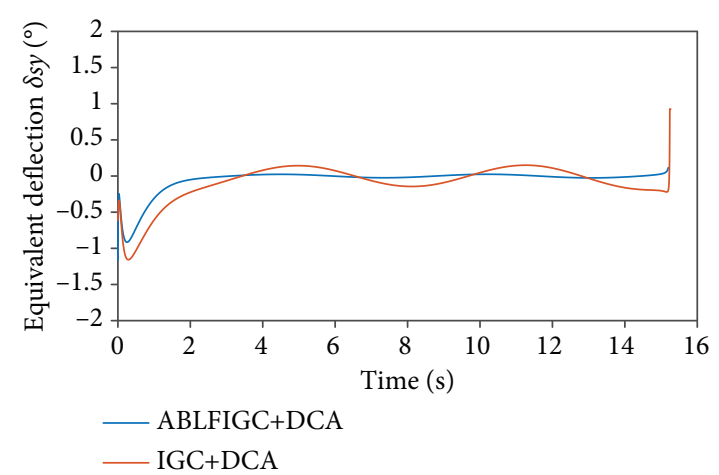

FIgURE 17: Curves of equivalent rudder deflection angle $\delta_{s y}$ : ABLFIGC+DCA refers to a combination of the proposed asymmetric barrier Lyapunov function-based IGC algorithm and the dynamic control allocation algorithm; IGC+DCA refers to a combination of the IGC algorithm subject to the traditional dynamic surface sliding-mode control law and the dynamic control allocation algorithm.

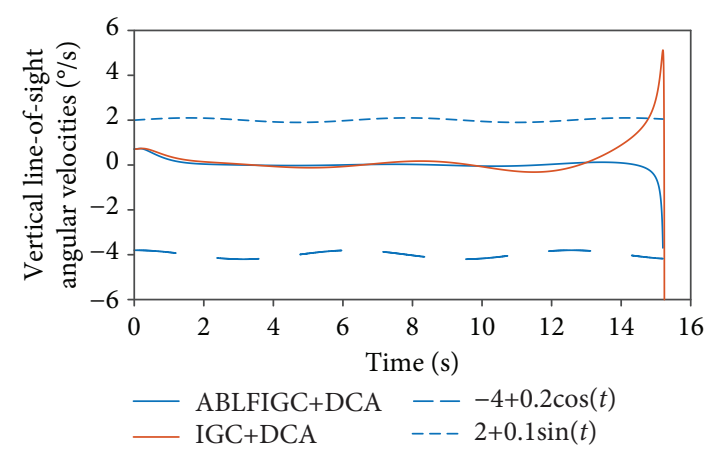

FIgURE 18: Curves of angular velocities of vertical line of sight of the interceptor missile in Scenario 1: ABLFIGC+DCA refers to a combination of the proposed asymmetric barrier Lyapunov function-based IGC algorithm and the dynamic control allocation algorithm; IGC+DCA refers to a combination of the IGC algorithm subject to the traditional dynamic surface sliding-mode control law and the dynamic control allocation algorithm. 


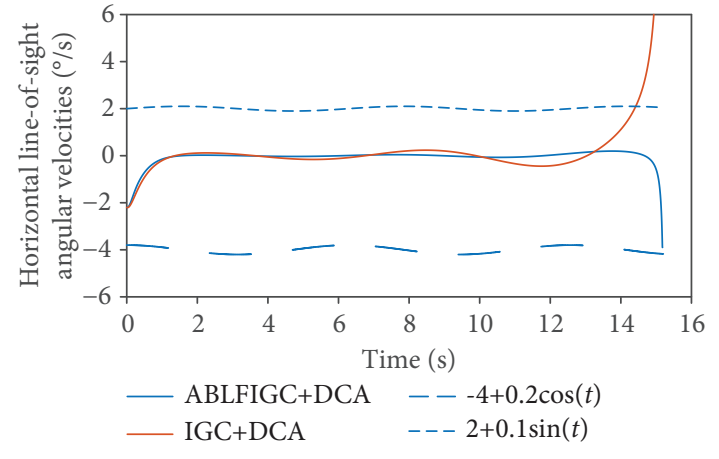

FIGURE 19: Curves of angular velocities of horizontal line of sight of the interceptor missile in Scenario 1: ABLFIGC+DCA refers to a combination of the proposed asymmetric barrier Lyapunov function-based IGC algorithm and the dynamic control allocation algorithm; IGC+DCA refers to a combination of the IGC algorithm subject to the traditional dynamic surface sliding-mode control law and the dynamic control allocation algorithm.

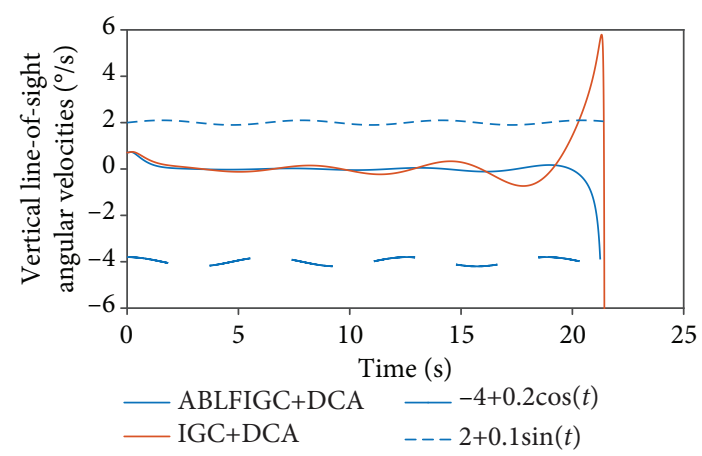

FIGURE 20: Curves of angular velocities of vertical line of sight of the interceptor missile in Scenario 2: ABLFIGC+DCA refers to a combination of the proposed asymmetric barrier Lyapunov function-based IGC algorithm and the dynamic control allocation algorithm; IGC+DCA refers to a combination of the IGC algorithm subject to the traditional dynamic surface sliding-mode control law and the dynamic control allocation algorithm.

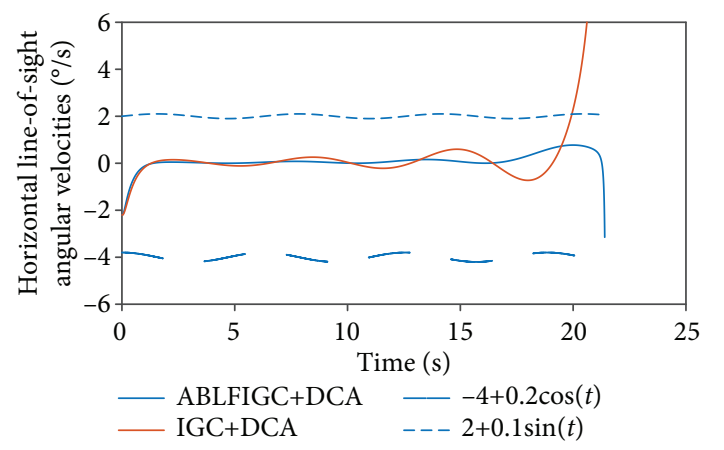

FIGURE 21: Curves of angular velocities of horizontal line of sight of the interceptor missile in Scenario 2: ABLFIGC+DCA refers to a combination of the proposed asymmetric barrier Lyapunov function-based IGC algorithm and the dynamic control allocation algorithm; IGC+DCA refers to a combination of the IGC algorithm subject to the traditional dynamic surface sliding-mode control law and the dynamic control allocation algorithm. problems of the system. This study combined the IGC method with an asymmetric barrier Lyapunov function to design an asymmetric barrier Lyapunov function-based IGC algorithm for the interceptor missile. Compared with traditional algorithms, by adopting the asymmetric barrier Lyapunov function, the proposed algorithm relaxed the constraints on the initial conditions of the system. In addition, the study utilized the dynamic surface sliding-mode control law and a TVGESO to design an IGC algorithm for interceptor missile, which not only reduced the system's requirements for high-order differentiability of the stability function but also enhanced the control system's resistance to unknown interferences. The study also considered the existing redundancy of the aerodynamic rudder and the reaction jet device in the actuator and consequently designed a dynamic control allocation algorithm to allocate the desired control moments to the actuator, thereby improving its efficiency. Finally, the simulation results showed that the proposed algorithm demonstrated relatively good dynamic characteristics and was able to satisfy the interceptor missile IGC system's requirements for accuracy and stability without breaking the constraints.

\section{Data Availability}

The simulation data used to support the findings of this study were supplied by Northwestern Polytechnical University under license and so cannot be made freely available. Requests for access to these data should be made to Prof. Xiaogeng Liang, E-mail: lxg2288@vip.sohu.com.

\section{Conflicts of Interest}

The authors declare that there is no conflict of interest regarding the publication of this paper.

\section{Acknowledgments}

This work was supported by the Aeronautical Science Foundation of China (grant number 2016ZC12005). The authors gratefully acknowledge the suggestions and help by the Prof. Xiaogeng Liang and Northwestern Polytechnical University.

\section{References}

[1] P. K. Menon and E. J. Ohlmeyer, "Integrated design of agile missile guidance and autopilot systems," Control Engineering Practice, vol. 9, no. 10, pp. 1095-1106, 2001.

[2] W. C. Xue, C. D. Huang, and Y. Huang, "Design methods for the integrated guidance and control system," Control Theory \& Applications, vol. 30, no. 12, pp. 1511-1520, 2013.

[3] Y. B. Shtessel and C. H. Tournes, "Integrated higher-order sliding mode guidance and autopilot for dual control missiles," Journal of Guidance, Control, and Dynamics, vol. 32, no. 1, pp. 79-94, 2009.

[4] M. Cross and Y. B. Shtessel, "Integrated guidance navigation and control using high-order sliding mode control for a missile interceptor," in AIAA Guidance, Navigation and Control Conference, pp. 1-17, Kissimmee, FL, USA, 2018. 
[5] X. L. Liang, M. Z. Hou, and G. R. Duan, "Integrated guidance and control for missile in the presence of input saturation and angular constraints," in Proceedings of the 32nd Chinese Control Conference, pp. 1070-1075, Piscataway, NJ, USA, 2013.

[6] X. Liang, M. Hou, and G. Duan, "Adaptive dynamic surface control for integrated missile guidance and autopilot in the presence of input saturation," Journal of Aerospace Engineering, vol. 28, no. 5, pp. 04014121-04014412, 2015.

[7] M. Hou, X. Liang, and G. Duan, "Adaptive block dynamic surface control for integrated missile guidance and autopilot," Chinese Journal of Aeronautics, vol. 26, no. 3, pp. 741-750, 2013.

[8] D. Zhou, P. Qu, and S. Sun, "A guidance law with terminal impact angle constraint accounting for missile autopilot," Journal of Dynamic Systems, Measurement, and Control, vol. 135, no. 5, pp. 051009-051010, 2013.

[9] C. Gao, C. Jiang, Y. Zhang, and W. Jing, “Three-dimensional integrated guidance and control for near space interceptor based on robust adaptive backstepping approach," International Journal of Aerospace Engineering, vol. 2016, Article ID 6598983, 11 pages, 2016

[10] S. Shamaghdari, S. K. Y. Nikravesh, and M. Haeri, "Integrated guidance and control of elastic flight vehicle based on robust MPC," International Journal of Robust and Nonlinear Control, vol. 25, no. 15, pp. 2608-2630, 2015.

[11] P. Bhavnesh, K. Subramanian, and S. E. Talole, "Robust integrated guidance and control design for tactical missiles," in AIAA Guidance, Navigation and Control Conference, pp. 1-20, Kissimmee, FL, USA, 2018.

[12] S. H. Seyedipour, M. Fathi Jegarkandi, and S. Shamaghdari, "Nonlinear integrated guidance and control based on adaptive back-stepping scheme," Aircraft Engineering and Aerospace Technology, vol. 89, no. 3, pp. 415-424, 2017.

[13] M. Fathi Jegarkandi, A. Ashrafifar, and R. Mohsenipour, "Adaptive integrated guidance and fault tolerant control using backstepping and sliding mode," International Journal of Aerospace Engineering, vol. 2015, Article ID 253478, 7 pages, 2015.

[14] H. Zhou, H. Zhao, H. Huang, and X. Zhao, "Integrated guidance and control design of the suicide UCAV for terminal attack," Journal of Systems Engineering and Electronics, vol. 28, no. 3, pp. 546-555, 2017.

[15] S. H. Viswanathan, A. K. Sanyal, and M. Izadi, "Integrated guidance and nonlinear feedback control of underactuated unmanned aerial vehicles in SE(3)," in AIAA Guidance, Navigation, and Control Conference, pp. 1-12, Grapevine, TX, USA, 2017.

[16] X. Wang, Y. Zheng, and H. Lin, "Integrated guidance and control law for cooperative attack of multiple missiles," Aerospace Science and Technology, vol. 42, pp. 1-11, 2015.

[17] S. Vaddi, P. K. Menon, and E. J. Ohlmeyer, "Numerical statedependent riccati equation approach for missile integrated guidance control," Journal of Guidance, Control, and Dynamics, vol. 32, no. 2, pp. 699-703, 2009.

[18] B. Panchal, N. Mate, and S. E. Talole, "Continuous-time predictive control-based integrated guidance and control," Journal of Guidance, Control, and Dynamics, vol. 40, no. 7, pp. 1579-1595, 2017.

[19] B. Niu, H. Li, Z. Zhang, J. Li, T. Hayat, and F. E. Alsaadi, "Adaptive neural-network-based dynamic surface control for stochastic interconnected nonlinear nonstrict-feedback systems with dead zone," IEEE Transactions on Systems, Man, and Cybernetics: Systems, pp. 1-13, 2018.

[20] B. Niu, D. Wang, N. D. Alotaibi, and F. E. Alsaadi, “Adaptive neural state-feedback tracking control of stochastic nonlinear switched systems: an average dwell-time method," IEEE Transactions on Neural Networks and Learning Systems, vol. 30, no. 4, pp. 1076-1087, 2019.

[21] R. E. Precup and H. Hellendoorn, "A survey on industrial applications of fuzzy control," Computers in Industry, vol. 62, no. 3, pp. 213-226, 2011.

[22] B. Niu, D. Wang, H. Li, X. Xie, N. D. Alotaibi, and F. E. Alsaadi, "A novel neural-network-based adaptive control scheme for output-constrained stochastic switched nonlinear systems," IEEE Transactions on Systems, Man, and Cybernetics: Systems, vol. 49, no. 2, pp. 418-432, 2019.

[23] K. B. Ngo, R. Mahony, and Z. P. Jiang, "Integrator backstepping using barrier functions for systems with multiple state constraints," in 44th IEEE Conference on Decision and Control, and the European Control Conference 2005, pp. 8306-8312, Seville, Spain, 2017.

[24] W. He and S. S. Ge, "Cooperative control of a nonuniform gantry crane with constrained tension," Automatica, vol. 66, pp. 146-154, 2016.

[25] W. He and Y. Dong, "Adaptive fuzzy neural network control for a constrained robot using impedance learning," IEEE Transactions on Neural Networks and Learning Systems, vol. 29, no. 4, pp. 1174-1186, 2018.

[26] B. Ren, S. S. Ge, K. P. Tee, and T. H. Lee, "Adaptive neural control for output feedback nonlinear systems using a barrier Lyapunov function," Neural Networks, vol. 21, no. 8, pp. 1339-1345, 2010.

[27] I. Salgado, M. Mera, and I. Chairez, "Suboptimal adaptive control of dynamic systems with state constraints based on barrier Lyapunov functions," IET Control Theory \& Applications, vol. 12, no. 8, pp. 1116-1124, 2018.

[28] K. P. Tee, B. Ren, and S. S. Ge, "Control of nonlinear systems with time-varying output constraints," Automatica, vol. 47, no. 11, pp. 2511-2516, 2011.

[29] B. Niu and J. Zhao, "Barrier Lyapunov functions for the output tracking control of constrained nonlinear switched systems," Systems and Control Letters, vol. 62, no. 10, pp. 963-971, 2013.

[30] Y. Qiu, X. Liang, and Z. Dai, "Backstepping dynamic surface control for an anti-skid braking system," Control Engineering Practice, vol. 42, pp. 140-152, 2015.

[31] Z. Dai, J. Cao, Y. Qiu, Y. Q. Chen, and X. Liang, “Backstepping dynamic surface control for a class of non-linear systems with time-varying output constraints," IET Control Theory \& Applications, vol. 9, no. 15, pp. 2312-2319, 2015.

[32] Y. Qiu, Z. Dai, Y. Chen, and X. Liang, "Constrained control for brushless DC motors with fractional friction compensation," in International Design Engineering Technical Conferences, Boston, MA, USA, 2015.

[33] X. Chen, Z. Dai, H. Lin, Y. Qiu, and X. Liang, "Asymmetric barrier Lyapunov function-based wheel slip control for antilock braking system," International Journal of Aerospace Engineering, vol. 2015, Article ID 917807, 10 pages, 2015.

[34] B. Xu, Z. Shi, F. Sun, and W. He, "Barrier Lyapunov function based learning control of hypersonic flight vehicle with AOA constraint and actuator faults," IEEE Transactions on Cybernetics, vol. 99, pp. 1-11, 2018. 
[35] H. An, H. Xia, and C. Wang, "Barrier Lyapunov functionbased adaptive control for hypersonic flight vehicles," Nonlinear Dynamics, vol. 88, no. 3, pp. 1833-1853, 2017.

[36] X. Lu, H. Zhao, B. Zhao, and J. Zhou, "Disturbance compensation-based integrated guidance and control design for near space interceptor," Control and Decision, vol. 32, no. 10, pp. 1782-1788, 2017.

[37] D. Swaroop, J. K. Hedrick, P. P. Yip, and J. C. Gerdes, "Dynamic surface control for a class of nonlinear systems," IEEE Transactions on Automatic Control, vol. 45, no. 10, pp. 1893-1899, 2000.

[38] T. A. Johansen and T. I. Fossen, "Control allocation-a survey," Automatica, vol. 49, no. 5, pp. 1087-1103, 2013. 


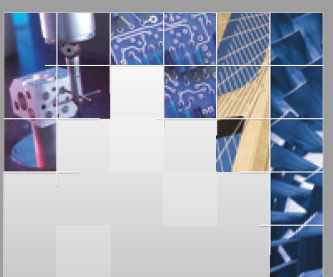

\section{Enfincering}
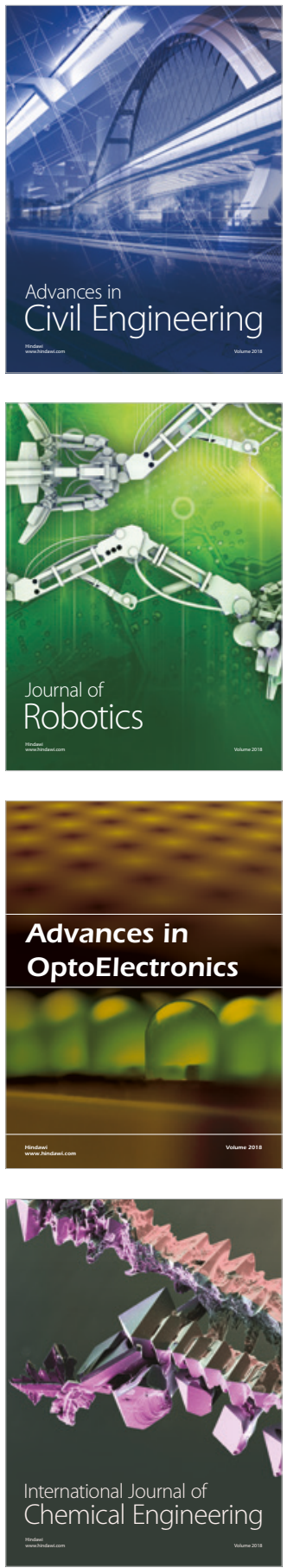

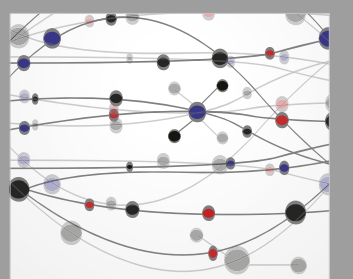

\section{Rotating \\ Machinery}

The Scientific World Journal

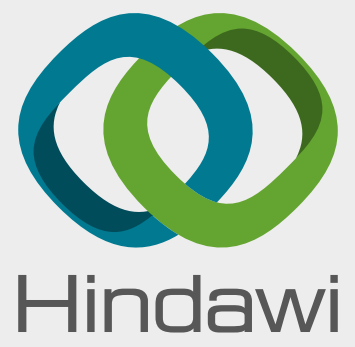

Submit your manuscripts at

www.hindawi.com
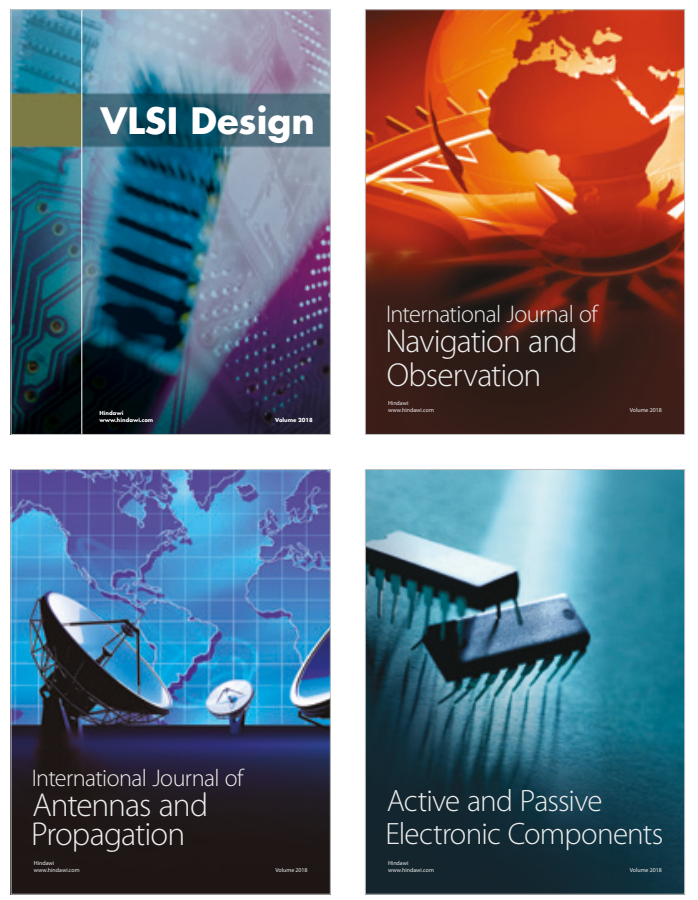
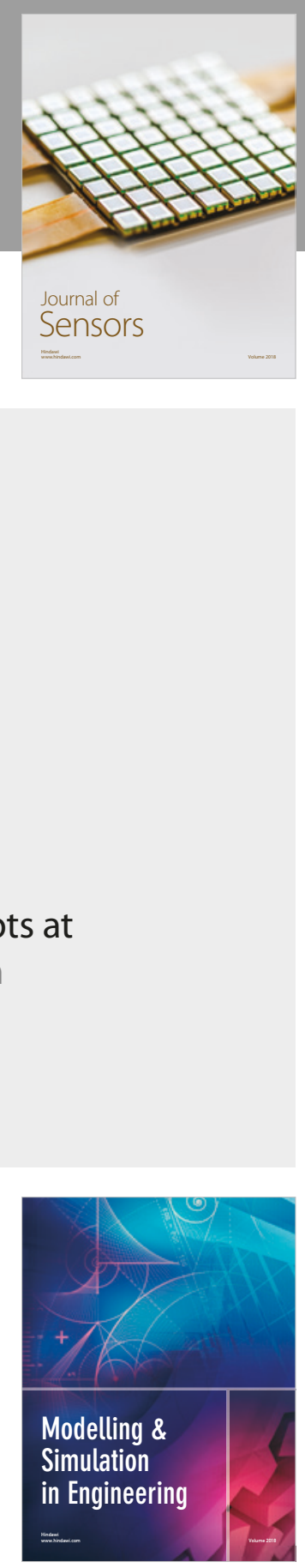

\section{Advances \\ Multimedia}
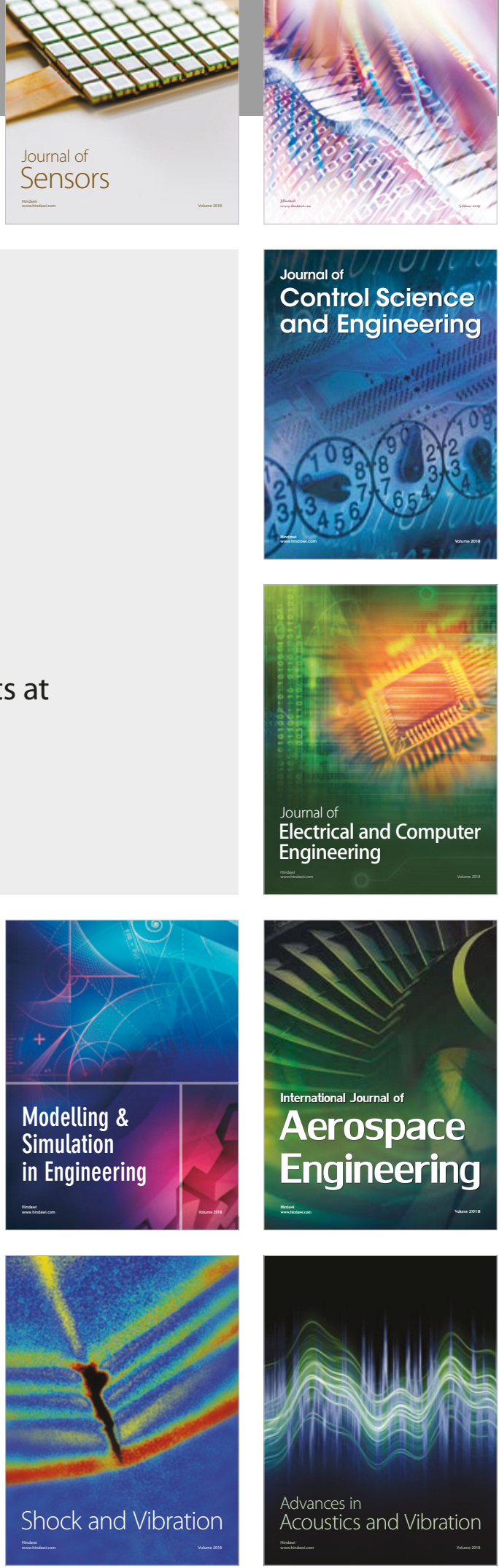